\title{
Liste commentée des Cleroidea (Coleoptera) de Suisse
}

\author{
Annotated checklist of Cleroidea (Coleoptera) of Switzerland \\ Yannick Chittaro $^{1}$, Andreas Sanchez ${ }^{1}$ \\ 1 info fauna-CSCF, Avenue Bellevaux 51, CH-2000 Neuchâtel, Switzerland \\ http://zoobank.org/CDCDBAD3-6E46-4243-850F-B3CA3F23EAA2 \\ Corresponding author: Yannick Chittaro (yannick.chittaro@unine.ch)
}

\begin{abstract}
Résumé
Received 8 May 2019

Accepted 21 June 2019

Published 10 July 2019

Academic editor:

Christian Monnerat

Une liste actualisée des espèces suisses appartenant à la superfamille des Cleroidea est présentée et brièvement commentée. La présence de 106 espèces de Biphyllidae (2 espèces), Byturidae (2), Cleridae (18), Melyridae (66), Phloiophilidae (1), Rhadalidae (8) et Trogossitidae (9) est attestée en Suisse sur la base de 18'989 occurrences issues de l'identification de spécimens de musées et de collections privées, ainsi que de la littérature. En parallèle, 37 espèces annoncées de Suisse par le passé sont exclues de la liste car insuffisamment documentées.
\end{abstract}

\section{Key Words}

species list

Switzerland

faunistics

distribution

\begin{abstract}
An updated checklist of the Swiss species belonging to the superfamily Cleroidea is presented and briefly discussed. A total of 106 species belonging to the families Biphyllidae (2 species), Byturidae (2), Cleridae (18), Melyridae (66), Phloiophilidae (1), Rhadalidae (8) and Trogossitidae (9) is confirmed for Switzerland. This list is based on 18'989 records obtained from the identification of specimens held in museum and private collections, and the literature. In parallel, 37 species that were recorded from Switzerland in the past are excluded from this list, for reasons of insufficient documentation.
\end{abstract}

\section{Introduction}

Plusieurs travaux de phylogénie moléculaire ont récemment bouleversé la classification supérieure traditionelle des Cleroidea telle que définie par Crowson (1955). Ainsi, les Biphyllidae et les Byturidae appartiennent maintenant à cette superfamille selon les conclusions de Hunt et al. (2007). Les Rhadalidae, précédemment traités comme une sous-famille des Dasytidae, sont maintenant considérés comme une famille à part entière suite au travail de Bocáková et al. (2012). Enfin, Gimmel et al. (2019), parmi d'autres propositions de changements, divisent les Trogossitidae en plusieurs familles (Lophocateridae, Peltidae, Thymalidae et Trogossitidae).

A l'échelle suisse, les Cleridae, les Melyridae et les Rhadalidae ont fait l'objet d'un travail très complet réalisé par Victor Allenspach, Robert Constantin et
Walter Wittmer (Allenspach et Wittmer 1979). Les autres familles n'ont par contre plus fait l'objet de listes nationales actualisées depuis les publications de Stierlin et Gautard (1867) et de Stierlin (1900), alors que la découverte en Suisse du seul représentant de la famille des Phloiophilidae, endémique d'Europe, date de 1992 seulement.

Compte tenu de l'évolution des connaissances systématiques et chorologiques au cours des quarante dernières années, une mise à jour de la liste faunistique des Cleroidea de Suisse s'avérait nécessaire. Basé sur un important travail de révision des collections suisses et sur une analyse critique de la littérature, cet article propose une liste commentée actualisée de l'ensemble des espèces de Cleroidea signalées de Suisse. Les espèces indigènes sont distinguées des espèces annoncées par erreur, insuffisamment documentées ou importées. 


\section{Matériel et méthode}

Afin de disposer de l'ensemble de l'information disponible et être ainsi à même d'évaluer au mieux l'appartenance des espèces à la faune de Suisse, nous avons procédé à un relevé exhaustif de l'ensemble du matériel des principales collections muséales suisses. Les collections des institutions suivantes ont donc été consultées et relevées dans leur intégralité (les noms des personnes de contact sont indiqués entre parenthèses)

AGRO Agroscope-Changins (anciennement SFRA), Nyon (Stève Breitenmoser)

BNM Bündner Natur-Museum, Chur (Stephan Liersch)

ETH Eidgenössische-Technische Hochschule, Zürich (Rod Eastwood, Michael Greeff)

KMLI Archäologie und Museum Baselland, Liestal (Marc Limat)

MHNF Musée d'histoire naturelle de Fribourg (Peter Wandeler)

MHNG Muséum d'histoire naturelle de Genève (Giulio Cuccodoro)

MHNN Musée d'histoire naturelle de Neuchâtel (Jessica Litman)

MHNS Musée de la nature du Valais, Sion (Nicolas Kramar, Sonja Gerber)

MSNL Museo cantonale di storia naturale, Lugano (Lucia Pollini, Michele Abderhalden)

MZA Museum zu Allerheiligen, Schaffhausen (Urs Weibel)

MZL Musée cantonal de zoologie, Lausanne (Anne Freitag)

NMAA Naturama, Aarau (Janine Mazenauer)

NMB Naturhistorisches Museum Basel (Matthias Borer)

NMBE Naturhistorisches Museum der Burgergemeinde Bern (Hannes Baur)

NMLU Natur-Museum, Luzern (Marco Bernasconi, Peter Herger)

NMTG Naturmuseum Thurgau, Frauenfeld (Barbara Richner)

NMSG Naturmuseum, St. Gallen (Priska Seri)

NMSO Naturmuseum, Solothurn (Christoph Germann, Marc Neumann)

SPZH Schädlingsprävention und -beratung, Zürich (Marcus Schmidt, Gabi Müller, Isabelle Landau-Lüscher)

Les collections privées des personnes suivantes ont également été prises en compte: Sylvie Barbalat (Neuchâtel NE), Mickaël Blanc (F-Sciez), Hansjörg Brägger (Bischofszell TG), Stève Breitenmoser (Givrins VD), Marie-Christine et Yannick Chittaro (Conthey VS), Vivien Cosandey (Essertines-sur-Rolle VD), Adrienne Frei (Zürich ZH), Michael Gilgen et Lea Kamber (Bangerten bei Dieterswil BE), Roman Graf (Horw LU), René Hoess (Bern BE), Barbara Huber (Thusis GR), Laurent Juillerat (Chézard-St-Martin NE), Wilfried Lö- derbusch (D-Markdorf), Christian Monnerat (Neuchâtel NE), Andreas Sanchez (Sion VS), Alexander Szallies (Wädenswil ZH) et Arnaud Vallat (Neuchâtel NE).

L'ensemble des données disponibles dans la littérature suisse a également été compilé. Les références des publications consultées sont intégralement mentionnées dans la bibliographie. Celles qui ne sont pas citées dans le texte de cet article sont signalées par un astérisque $\left(^{*}\right)$.

Les ouvrages et articles suivants ont été utilisés pour l'identification des espèces: Pardo Alcaide (1962), Freude et al. (1967, 1979), Kolibáč (1992, 1993, 2018), Lohse et Lucht (1992), Gerstmeier (1998), Kolibáč et al. (2005), Liberti et Focarile (2005), Plata Negrache (2009, 2012), Constantin et Liberti (2011) et Niehuis (2013).

Sans indication explicite, les informations générales sur la distribution des espèces sont tirées du «Catalogue of Palaearctic Coleoptera » édité par Löbl et Smetana (2007), qui n'est alors pas cité dans les textes consacrés aux espèces. Les ouvrages utilisés pour l'identification ont également fourni de précieuses informations sur la répartition des espèces, de même que les publications de Callot (2018) pour l'Alsace, de Tronquet (2014) pour la France, de Brandstetter et Kapp (1998) pour le Liechtenstein et le Vorarlberg autrichien, de Köhler et Klausnitzer (1998) et de Köhler $(2000,2011)$ pour l'Allemagne et de Kahlen et Hellrigl (1996) pour le Sud-Tyrol italien.

La nomenclature et systématique suivies sont celles $\mathrm{du}$ «Catalogue of Palaearctic Coleoptera» pour les espèces (Jelínek 2007, Kolibáč 2007, Löbl 2007, Löbl et al. 2007, Mayor 2007a, b). Par rapport à ce document de référence, nous avons néanmoins considéré les exceptions suivantes:

- nous avons pris en compte la description récente du genre Anthomalachius pour les espèces du groupe «Clanoptilus spinosus » (Tshernyshev 2009),

- nous avons suivi la mise en synonymie de Danacea serbica Kiesenwetter, 1863 avec $D$. iners Kiesenwetter, 1859 par Liberti (2009a),

- de même, nous avons privilégié Dasytes gonocerus Mulsant \& Rey, 1868 à D. erratus Schilsky, 1895 suite au travail de synonymisation réalisé par Liberti et Constantin (2011),

- enfin, nous avons suivi les choix de Liberti et Constantin (2009) à propos du genre Enicopus Stephens, 1830, et avons donc considéré E. ater ater (Fabricius, 1787) comme distinct d'E. pilosus (Scopoli, 1763).

La liste des principaux synonymes de chaque taxon est détaillée dans le " Catalogue of Palaearctic Coleoptera » auquel on se réfèrera.

Pour la classification supérieure, nous avons suivi Bouchard et al. (2011), en considérant néanmoins les Rhadalidae comme famille indépendante (Bocáková et al. 2012, Gimmel et al. 2019), ainsi que les Biphyllidae et les Byturidae comme faisant partie des Cleroidea (Hunt et al. 2007, Bocák et al. 2014, Gimmel et al. 2019). Nous n'avons par contre pas suivi la récente division en 
plusieurs familles des traditionnels Trogossitidae, comme proposé par Gimmel et al. (2019), dans l'attente de confirmation de leurs résultats.

Pour les plantes et champignons cités dans le document, nous avons suivi les choix taxonomiques respectifs de Juillerat et al. (2017) et de la base de donnée nationale SwissFungi (swissfungi.wsl.ch).

Une fois l'ensemble de l'information disponible, nous avons suivi la procédure proposée par Monnerat et al. (2015a) pour statuer sur l'indigénat des espèces en Suisse. Ainsi, nous n'avons pas retenu une espèce si les données relatives ne satisfaisaient pas au minimum jugé nécessaire (étiquetage univoque, collections fiables, ...).

Les espèces suivies d'une lettre et d'un chiffre en gras ( C1 » par exemple) font l'objet d'un commentaire. Ainsi, un commentaire est fourni pour les espèces les plus rares (connues de moins de vingt occurrences valides en Suisse) et l'ensemble des spécimens examinés et toutes les observations publiées sont mentionnés afin de documenter et de justifier leur prise en compte. Certaines espèces ne font l'objet que de commentaires d'ordre taxonomique ou chorologique. Tout le matériel examiné a été déterminé ou revu par les auteurs ou par des spécialistes européens (voir remerciements).

Les spécimens examinés et les données issues de la littérature sont présentés par ordre chronologique de découverte ou de publication, puis par ordre alphabétique des localités en fonction des informations disponibles. Toutes les occurrences sont citées sur le schéma suivant : nombre d'exemplaires, localité pour les données anciennes ou commune et canton abrégé pour les données récentes (à partir de l'an 2000), date, collectionneur, déterminateur, collection, acronyme officiel du musée où l'insecte est déposé lorsque cela est le cas.

Les informations qui concernent la localité et la date sont indiquées telles qu'elles figurent sur l'étiquette. Les interprétations des abréviations alphabétiques sont mentionnées entre crochets « [ ] ». Dans les collections anciennes, le collectionneur (leg.) n'est pas toujours mentionné textuellement sur les étiquettes. Nous avons donc généralement préféré la mention « coll. » jugée plus adéquate. Dans de nombreux cas, le nom du détenteur de la collection ne figure pas sur les étiquettes. Néanmoins, en fonction de l'expérience acquise dans les relevés de collections, l'attribution de certains types d'étiquettes et/ ou d'écritures à une collection particulière s'est souvent avérée possible.

La collection de Charles Maerky, déposée au Muséum d'histoire naturelle de Genève, est considérée depuis longtemps comme problématique (Monnerat et al. 2015a). Mis à part les individus provenant de sa collection (« coll. Maerky C. »), elle contient également des insectes issus d'autres collections (mentionnées alors " ex coll. Melly A. » par exemple) et pour lesquelles les étiquettes originales font défaut. De ce fait, et afin de ne pas perdre l'information quant au fait que ces insectes appartiennent à la collection de Maerky C., nous avons retenu la mention de « coll. Maerky C. » pour l'intégralité de sa collection.
Pour les données issues de la littérature, détaillées sous « Données publiées », nous avons retenu la localité telle que mentionnée dans la citation originale. La personne " source » (et non pas le legit) est considérée comme l'auteur dans la publication et mentionnée par exemple comme suit: "Ormontsthal par Venetz I. (Stierlin et Gautard 1867) ». Lorsque la même localité est citée à plusieurs reprises, seule la plus ancienne est retenue ici, les localités annoncées dans les publications ultérieures étant généralement reprises telles quelles et parfois même tronquées. Nous nous sommes néanmoins dispensés de mentionner ici toutes les données figurant dans Allenspach et Wittmer (1979) si nous avons retrouvé et vérifié l'identification des spécimens qu'ils citent, et qui figurent alors sous « Matériel examiné ». Ne subsistent donc que les données d'Allenspach et Wittmer (1979) pour lesquelles nous n'avons pas retrouvé le matériel correspondant ou lorsqu'il s'agissait d'identification erronées que nous avons corrigées.

Parmi les données citées dans le document, que ce soit sous « Matériel examiné » ou sous « Données publiées », celles que nous considérons comme insuffisantes pour être retenues sont précédées d'un nombre, noté comme suit ${ }^{11}{ }^{1)}$, qui fait référence aux différentes catégories de sources d'erreurs identifiées et détaillées dans Monnerat et al. (2015a), à savoir :

1) données sources invérifiables

2) déterminations erronées

3) spécimens issus de collections problématiques

4) spécimens d'origine inconnue attribués à des localités suisses

5) double étiquetage, interprétation ou recopie erronées du nom de la localité d'origine

6) confusion entre localité d'origine, lieu d'élevage ou d'éclosion des spécimens et de dépôt de la collection

7) localités non suisses ou localités étrangères homonymes

8) incohérences chorologiques ou écologiques

Autres abréviations utilisées: coll $=$ collection, det. $=$ déterminateur, ex. $=$ exemplaire, leg. $=$ collectionneur, s.l.= sensu lato (sens large), cantons suisses abrégés $(\mathrm{AG}=\mathrm{Ar}-$ govie, $\mathrm{BE}=$ Berne, $\mathrm{BS}=\mathrm{Bâle}-\mathrm{Ville}, \mathrm{BL}=$ Bâle-Campagne, $\mathrm{GE}=$ Genève, $\mathrm{GR}=$ Grisons, LU=Lucerne, $\mathrm{NE}=\mathrm{Neu}-$ châtel, $\mathrm{SG}=$ Saint-Gall, $\mathrm{SH}=$ Schaffhouse, $\mathrm{SO}=$ Soleure, $\mathrm{TI}=$ Tessin, $\mathrm{TG}=$ Thurgovie, $\mathrm{VD}=$ Vaud, $\mathrm{VS}=$ Valais, $\mathrm{ZH}=$ Zurich).

\section{Résultats}

\section{Liste des Cleroidea de Suisse}

Les auteurs considèrent que les 106 espèces mentionnés en gras dans cette liste forment ou ont formé des populations en Suisse, même si pour bon nombre d'entre elles les informations disponibles sont rares et ponctuelles.

Les 37 espèces indiquées entre crochets « [ ] » et décalées dans la liste ne doivent par contre pas être considérées comme appartenant à la faune suisse, tout du moins 
jusqu'à ce que de nouvelles données viennent infirmer notre opinion. Sont associées à cette catégorie des espèces dont les individus de référence émanent de collections problématiques, telles que celles de Charles Maerky ou de Max Täschler (Monnerat et al. 2015a), mentionnées de Suisse par erreur suite à des identifications erronées, citées dans des publications anciennes comme celle de Stierlin et Gautard (1867) sans individus de référence et considérées comme douteuses, ou encore des espèces importées. Certaines sont potentielles pour la Suisse, mais leur indigénat reste à confirmer, les données disponibles n'étant pas suffisantes à l'heure actuelle.

Afin de faciliter la recherche des espèces dans ce document, les taxa apparaissent dans l'ordre alphabétique des familles, des sous-familles, des genres, des sous-genres, des espèces et des sous-espèces.

L'ensemble des informations collectées représente un total de 18'989 occurrences pour les familles traitées. Les cartes de répartition actualisées de toutes les espèces retenues pour la Suisse sont disponibles sur le serveur cartographique d'info fauna - CSCF (Centre suisse de cartographie de la faune) (http://lepus.unine.ch/carto/). L'ensemble des données validées a également mis à disposition sur GBIF.org (https://doi.org/10.15468/dl.8up98w).

\section{BIPHYLLIDAE LeConte, 1861}

Biphyllus lunatus (Fabricius, 1787) C1

Diplocoelus fagi (Chevrolat, 1837)

BYTURIDAE Gistel, 1848

Byturinae Gistel, 1848

Byturus ochraceus (Scriba, 1790)

Byturus tomentosus (DeGeer, 1774)

CLERIDAE Latreille, 1802

Clerinae Latreille, 1802

Allonyx quadrimaculatus (Schaller, 1783) C2

Clerus mutillarius mutillarius Fabricius, 1775

Opilo domesticus (Sturm, 1837)

[Opilo germanus (Chevrolat, 1843)] C3

Opilo mollis (Linnaeus, 1758)

Opilo pallidus (A. G. Olivier, 1795) C4

Thanasimus femoralis (Zetterstedt, 1828)

Thanasimus formicarius formicarius (Linnaeus, 1758)

Trichodes alvearius (Fabricius, 1792)

Trichodes apiarius (Linnaeus, 1758)

[Trichodes favarius (Illiger, 1801)] C5

[Trichodes cf. heydeni Escherisch, 1892] C6

[Trichodes ircutensis (Laxmann, 1770)] C7

Korynetinae Laporte, 1836

Dermestoides sanguinicollis (Fabricius, 1787) C8

Korynetes caeruleus (DeGeer, 1775)

Korynetes ruficornis (Sturm, 1837)

Necrobia ruficollis (Fabricius, 1775)

Necrobia rufipes (DeGeer, 1775)

Necrobia violacea (Linnaeus, 1758)

[Tarsostenus univittatus (P. Rossi, 1792)] C9
Tillinae Fischer von Waldheim 1813

Denops albofasciatus (Charpentier, 1825) C10

Tilloidea unifasciata (Fabricius, 1787)

Tillus elongatus (Linnaeus, 1758)

\section{MELYRIDAE Leach, 1815}

Dasytinae Laporte, 1840

[Danacea (Allodanacaea) wittmeri Liberti, 1985] C11

Danacea (Danacea) ambigua Mulsant \& Rey, 1868

Danacea (Danacea) denticollis Baudi di Selve, 1861 [Danacea (Danacea) iners Kiesenwetter, 1859] C12

[Danacea (Danacea) marginata (Küster, 1851)] C13

Danacea (Danacea) montivaga Mulsant \& Rey, 1868 C14 [Danacea (Danacea) morosa Kiesenwetter, 1863] C15 [Danacea (Danacea) nigripalpis Fiori, 1912] C16

Danacea (Danacea) nigritarsis alpina Pic, 1894 C17

Danacea (Danacea) nigritarsis nigritarsis (Küster, 1850) $\mathrm{C} 17$

Danacea (Danacea) pallipes (Panzer, 1793)

Dasytes (Anthoxenus) subaeneus Schönherr, 1817 C18

[Dasytes (Dasytes) cf. murinus Schilsky, 1894] C19

Dasytes (Dasytes) niger (Linnaeus, 1761)

Dasytes (Dasytes) pauperculus Laporte, 1840 C20

Dasytes (Dasytes) thoracicus thoracicus Mulsant \& Rey, 1868 C21

Dasytes (Hypodasytes) alpigradus Kiesenwetter, 1863

Dasytes (Hypodasytes) gonocerus Mulsant \& Rey, 1868

Dasytes (Hypodasytes) lombardus Fiori, 1909 C22

Dasytes (Hypodasytes) obscurus Gyllenhal, 1813

Dasytes (Hypodasytes) subalpinus subalpinus Baudi di Selve, 1873

[Dasytes (Hypodasytes) tristiculus Mulsant \& Rey, 1868] C23

[Dasytes (Mesodasytes) aeneiventris Küster 1850] C24

Dasytes (Mesodasytes) aeratus Stephens, 1830

Dasytes (Mesodasytes) plumbeus (O. F. Müller, 1776)

Dasytes (Mesodasytes) virens (Marsham, 1802)

Dasytes (Metadasytes) caeruleus (DeGeer, 1774)

Dasytes (Metadasytes) fusculus (Illiger, 1801)

Dasytes (Metadasytes) nigrocyaneus Mulsant \& Rey, $1868 \mathrm{C} 25$

[Dasytidius nigripes (Pic, 1894)] C26

Divales bipustulatus (Fabricius, 1781)

[Divales quadrimaculatus (A. G. Olivier, 1790)] C27

Dolichosoma lineare (P. Rossi, 1794)

[Enicopus (Enicopus) ater ater (Fabricius, 1787)] C28

Enicopus (Enicopus) pilosus (Scopoli, 1763) C29

[Psilothrix (Psilothrix) aureola (Kiesenwetter, 1859)] C30

[Psilothrix (Psilothrix) smaragdina (P. H. Lucas, 1846)] C31

Psilothrix (Psilothrix) viridicoerulea (Geoffroy, 1785)

Malachiinae Fleming, 1821

Anthocomus (Anthocomus) fasciatus (Linnaeus, 1758)

Anthocomus (Anthocomus) rufus rufus (Herbst, 1784)

Anthocomus (Celidus) equestris (Fabricius, 1781)

Anthocomus (Celidus) humeralis (Morawitz, 1862) C32 
[Anthomalachius spinosus (Erichson, 1840)] C33

[Attalus (Abrinus) amictus (Erichson, 1840)] C34

Attalus (Abrinus) analis (Panzer, 1798)

Attalus (Antholinus) minimus (P. Rossi, 1790)

Attalus (Attalus) alpinus (Giraud, 1851) C35

[Attalus (Attalus) cyaneus (Fabricius, 1787)] C36

Axinotarsus (Axinotarsus) marginalis (Laporte, 1840)

Axinotarsus (Axinotarsus) pulicarius (Fabricius, 1777)

Axinotarsus (Axinotarsus) ruficollis (A. G. Olivier, 1790)

Cerapheles terminatus (Ménétriés, 1832) C37

[Ceratistes dilaticornis (Germar, 1824)] C38

Charopus concolor (Fabricius, 1801)

Charopus docilis Kiesenwetter, 1852 C39

Charopus flavipes (Paykull, 1798) C40

Charopus madidus Kiesenwetter, 1863 C41

Charopus pallipes (A. G. Olivier, 1790) C42

Clanoptilus (Clanoptilus) affinis (Ménétriés, 1832) C43

[Clanoptilus (Clanoptilus) arnaizi (Pardo Alcaide, 1966)] C44

Clanoptilus (Clanoptilus) elegans (A. G. Olivier, 1790)

Clanoptilus (Clanoptilus) emarginatus (Krauss, 1902)

Clanoptilus (Clanoptilus) geniculatus (Germar, 1824) C45

Clanoptilus (Clanoptilus) marginellus (A. G. Olivier, 1790)

[Clanoptilus (Clanoptilus) rufus (A. G. Olivier, 1790)]

C46

[Clanoptilus (Clanoptilus) spinipennis (Germar,

1824)] C47

[Clanoptilus (Hypoptilus) barnevillei (Puton, 1865)] C48

Cordylepherus viridis (Fabricius, 1787)

Cyrtosus (Oogynes) ovalis (Laporte, 1838) C49

[Ebaeus (Ebaeus) appendiculatus Erichson, 1840] C50

[Ebaeus (Ebaeus) ater Kiesenwetter, 1863] C51

Ebaeus (Ebaeus) battonii Pardo Alcaide, 1962 C52

[Ebaeus (Ebaeus) collaris collaris Erichson, 1840] C53

Ebaeus (Ebaeus) flavicornis Erichson, 1840 C54

Ebaeus (Ebaeus) gibbus (Drapiez, 1819) C55

Ebaeus (Ebaeus) pedicularius pedicularius (Linnaeus, 1758)

[Ebaeus (Ebaeus) cf. rufipes Morawitz, 1861] C56

Ebaeus (Ebaeus) thoracicus (Geoffroy, 1785)

Hypebaeus (Alloceps) albifrons albifrons (Fabricius, 1775)

[Hypebaeus (Hypebaeus) flavicollis (Erichson, 1840)] C57

Hypebaeus (Hypebaeus) flavipes (Fabricius, 1787)

Malachius (Malachius) aeneus (Linnaeus, 1758)

Malachius (Malachius) australis Mulsant \& Rey, 1867

Malachius (Malachius) bipustulatus (Linnaeus, 1758)

Malachius (Malachius) rubidus Erichson, 1840

Malachius (Malachius) scutellaris Erichson, 1840 C58

Micrinus heteromorphus (Abeille de Perrin, 1869) C59

Micrinus inornatus (Küster, 1846)

Nepachys cardiacae (Linnaeus, 1761) C60

Sphinginus coarctatus (Erichson, 1840) C61

Sphinginus lobatus lobatus (A. G. Olivier, 1790)

Troglops albicans (Linnaeus, 1767)
[Troglops cephalotes (A. G. Olivier, 1790)] C62

Troglops silo Erichson, 1840

PHLOIOPHILIDAE Kiesenwetter, 1863

Phloiophilus edwardsii Stephens, 1830 C63

RHADALIDAE LeConte, 1861

Rhadalinae LeConte, 1861

[Aplocnemus (Aplocnemus) albipilis (Kiesenwetter, 1863)] C64

Aplocnemus (Aplocnemus) alpestris (Kiesenwetter, 1861)

Aplocnemus (Aplocnemus) chalconatus (Germar, 1817) $\mathbf{C 6 5}$

Aplocnemus (Aplocnemus) impressus (Marsham, 1802)

Aplocnemus (Aplocnemus) integer (Baudi di Selve, 1873) $\mathrm{C} 66$

Aplocnemus (Aplocnemus) nigricornis nigricornis (Fabricius, 1792)

[Aplocnemus (Aplocnemus) ramicornis Kiesenwetter, 1863] C67

Aplocnemus (Aplocnemus) tarsalis (C. R. Sahlberg, 1822) Aplocnemus (Aplocnemus) virens virens (Suffrian, 1843)

[Trichoceble floralis (A. G. Olivier, 1790)] C68

Trichoceble memnonia (Kiesenwetter, 1861) C69

THANEROCLERIDAE Chapin, 1924

Thaneroclerinae Chapin, 1924

[Thaneroclerus buquet (Lefebvre, 1835)] C70

TROGOSSITIDAE Latreille 1802

Lophocaterinae Crowson, 1964

Grynocharis oblonga (Linnaeus, 1758) C71

Peltinae Latreille, 1806

Peltis ferruginea (Linnaeus, 1758)

Peltis grossa (Linnaeus, 1758)

Thymalus limbatus (Fabricius, 1787)

Trogossitinae Latreille 1802

Calitys scabra (Thunberg, 1784) C72

Nemozoma caucasicum Ménétriés, 1832 C73

[Nemozoma cornutum Sturm, 1826] C74

Nemozoma elongatum (Linnaeus, 1761)

Temnoscheila caerulea (A. G. Olivier, 1790)

Tenebroides mauritanicus (Linnaeus, 1758) C75

Commentaires sur quelques espèces

C1) Biphyllus lunatus

Matériel examiné: ${ }^{3)} 1$ ex., Genève, coll., Maerky C., ex coll. Melly A., MHNG; 1 ex., Bois de Chênes, 6.1994, leg. Besuchet C., MHNG; 1 ex., Cologny GE, 20.4.2010, leg. \& coll. Blanc M.

Données publiées. ${ }^{1)}$ Genf par Tournier H. et ${ }^{1)}$ Vevey par Gautard V. (Stierlin et Gautard 1867).

Commentaire. Assez largement répandue en Europe, cette espèce est pourtant généralement rare. Seuls deux 
spécimens, des cantons de Vaud et de Genève, attestent de sa présence en Suisse. L'espèce est inféodée au genre de pyrénomycètes Daldinia, surtout $D$. concentrica, qui pousse sur le frêne (Fraxinus excelsior) principalement.

\section{C2) Allonyx quadrimaculatus}

Fig. 1A

Matériel examiné. 1 ex., Helvet. [Helvetia], leg. Anonymous, ETH; ${ }^{3)} 1$ ex., Wülflingen, 6.1853, coll. Dietrich K., ETH; ${ }^{3)} 1$ ex., Wülflingen, 13.7.1858, coll. Dietrich K., ETH; 1 ex., Finges, 16.6.1962, leg. Besuchet C., MZL; 1 ex., Finges, 15.4.1979, leg. Besuchet C., MHNG; 1 ex., Finges, 6.1992, leg. Besuchet C., MHNG; 1 ex., Wallis, Pfinwald [Pfynwald], 27.6.1992, leg. \& coll. Gfeller W.; 1 ex., Leuk VS, 15.5.2002, [leg. Carron G.], MHNN; 2 ex., Anniviers VS, 5.-20.6.2014, 12.-26.5.2015, leg. \& coll. Sanchez A.; 12 ex., Visp VS, 6.5.-28.7.2016, leg. \& coll. Sanchez A.; 1 ex., Leuk VS, 24.5.-1.7.2018, leg. \& coll. Sanchez A. ; 1 ex., Riddes VS, 18.4.-22.5.2019, leg. \& coll. Sanchez A.; 1 ex., Martigny VS, 21.5.-5.6.2019, leg. \& coll. Sanchez A.

Données publiées. ${ }^{3}$ Wülflingen, 6.1853 et ${ }^{1)}$ Nürensdorf, 1858 par Dietrich K. (Dietrich 1865); ${ }^{1)} \mathrm{Genf}$ par Tournier H. et ${ }^{1)}$ Schaffhausen par Stierlin G. (Stierlin et Gautard 1867); Kanton Wallis, Susten, 27.6.1992 par Gfeller W. (Gfeller 1992); 1 ex., Anniviers VS, 5.20.6.2014 par Sanchez A. (Sanchez et al. 2015); nombreux ex., Visp VS, 6.5.-28.7.2016 par Sanchez A. (Chittaro et Sanchez 2017).

Commentaire. Assez largement répandue en Europe, cette espèce est pourtant rare en Suisse, où elle n'est connue que de quelques localités et d'un faible nombre d'individus. En 2016, de nombreux exemplaires ont néanmoins été observés chassant sur des troncs brûlés de pin sylvestre (Pinus sylvestris), dans une forêt incendiée cinq ans auparavant (Chittaro et Sanchez 2017).

\section{C3) [Opilo germanus]}

Données publiées. ${ }^{2,8)} 1$ ex., Lausanne VD, MZL (Allenspach et Wittmer 1979).

Commentaire. Si le statut taxonomique d'O. germanus est toujours incertain, la plupart des auteurs le considèrant comme synonyme d'O. abeillei Korge, 1960, d'O. domesticus ou d'O. mollis (Hubenthal 1916, Korge 1960, Kolibáč 2018, Gerstmeier R., comm. pers.), Tihelka (2016) l'annonce récemment comme nouveau pour l'Italie et fournit une synthèse des observations européennes du taxon. Il constate une répartition originale: toutes les données sont localisées à proximité de régions côtières, souvent de grands ports, et suggère dès lors que le taxon n'est peut-être pas indigène en Europe mais serait arrivé accidentellement au cours de différentes introductions. Nous avons retrouvé le spécimen cité dans la littérature suisse (Allenspach et Wittmer 1979) qui s'avère être, à notre avis, $O$. domesticus. Indépendamment des décisions concernant sa position taxonomique, $O$. germanus ne fait pas partie de notre faune.

\section{C4) Opilo pallidus}

Matériel examiné. ${ }^{3)} 1$ ex., Genf, coll. Lasserre H., ETH; ${ }^{3)} 1$ ex., Genf, coll. Linder A., ex coll. Täschler M., ETH; 3) 1 ex., Jura, Gimel, 2.8., leg. \& coll. Maerky C., MHNG; 1 ex., Châtelaine, 6.8.1950, leg. Simonet J., MHNG; 1 ex., Commugny VD, 13.7.1954, leg. Steffen J., MHNG; 1 ex., Mendrisio, 10.1955, leg. \& coll. Lautner J., NMB; 3 ex., Chiasso, 31.7.1967, leg. \& coll. Scherler P., NMBE; 2 ex., Chiasso, 14.7.1969, leg. \& coll. Scherler P., NMBE; 1 ex., Piazzogna, 6.1984, leg. \& coll. Ettmüller W.; 1 ex., Airela-Ville, 31.8.1988, leg. \& coll. Scherler P., NMBE; 1 ex., Genève, La Laire, 8.-9.1990, leg. Besuchet C., MHNG; 1 ex., Genève, Vandoeuvres, 8.-9.1990, leg. Besuchet C., MHNG; 1 ex., Genève, Lullier, 8.1991, leg. SFRA [Station fédérale de recherche agronomique], MHNG; 1 ex., Vaud, Nyon, 9.1991, leg. Besuchet C., MHNG; 1 ex., Genève, Avully, 8.1993, leg. Besuchet C., MHNG.

Données publiées. ${ }^{1)}$ Genf par Chevrier F., ${ }^{3)}$ Genf par Lasserre H. et ${ }^{1}$ Genf par Tournier H. (Stierlin et Gautard 1867); ${ }^{1)}$ Chiasso (Fontana 1947).

Commentaire. Quelques données des cantons de Genève, de Vaud et du Tessin attestent de la présence en Suisse de cette espèce plutôt méridionale, qui se développe dans la canopée de différentes essences feuillues, mais principalement les chênes (Quercus spp.) (Sanchez et al. 2018).

\section{C5) [Trichodes favarius]}

Données publiées. ${ }^{1,8}$ [Suisse] par Heer O. (Bremi-Wolf 1856); ${ }^{2,8} 1$ ex., Canton Tessin, ETH (Allenspach et Wittmer 1979).

Commentaire. La citation de Bremi-Wolf (1856) est invérifiable. Quant au spécimen du Tessin, il a été retrouvé et se rapporte en réalité à Trichodes cf. heydeni (voir C6). T. favarius est une espèce orientale et sa présence en Suisse est très peu probable.

\section{C6) [Trichodes cf. heydeni]}

Matériel examiné. ${ }^{4,5,8)} 1$ ex., Canton Tessin, leg. Anonymous, det. Gerstmeier R., coll. Allenspach V., NMB (ex coll. ETH).

Commentaire. Le seul spécimen examiné était cité dans la littérature sous T. favarius (Allenspach et Wittmer 1979) (voir C5). Après vérification de l'identification, l'individu se rapproche en réalité de T. heydeni, une espèce connue uniquement d'Azerbaidjan, d'Arménie, d'Iran, d'Iraq et de Turquie. S'agissant d'une femelle, l'identification spécifique de cet individu ne peut néanmoins être assurée. Le taxon est dans tous les cas absent de notre faune, et il s'agit vraisemblablement d'une erreur d'étiquetage du spécimen examiné.

\section{C7) [Trichodes ircutensis]}

Données publiées. ${ }^{1,8)} 1$ ex., bei Klosters, Graubünden, 7.1910 par Müller J.A. (Müller 1912). 


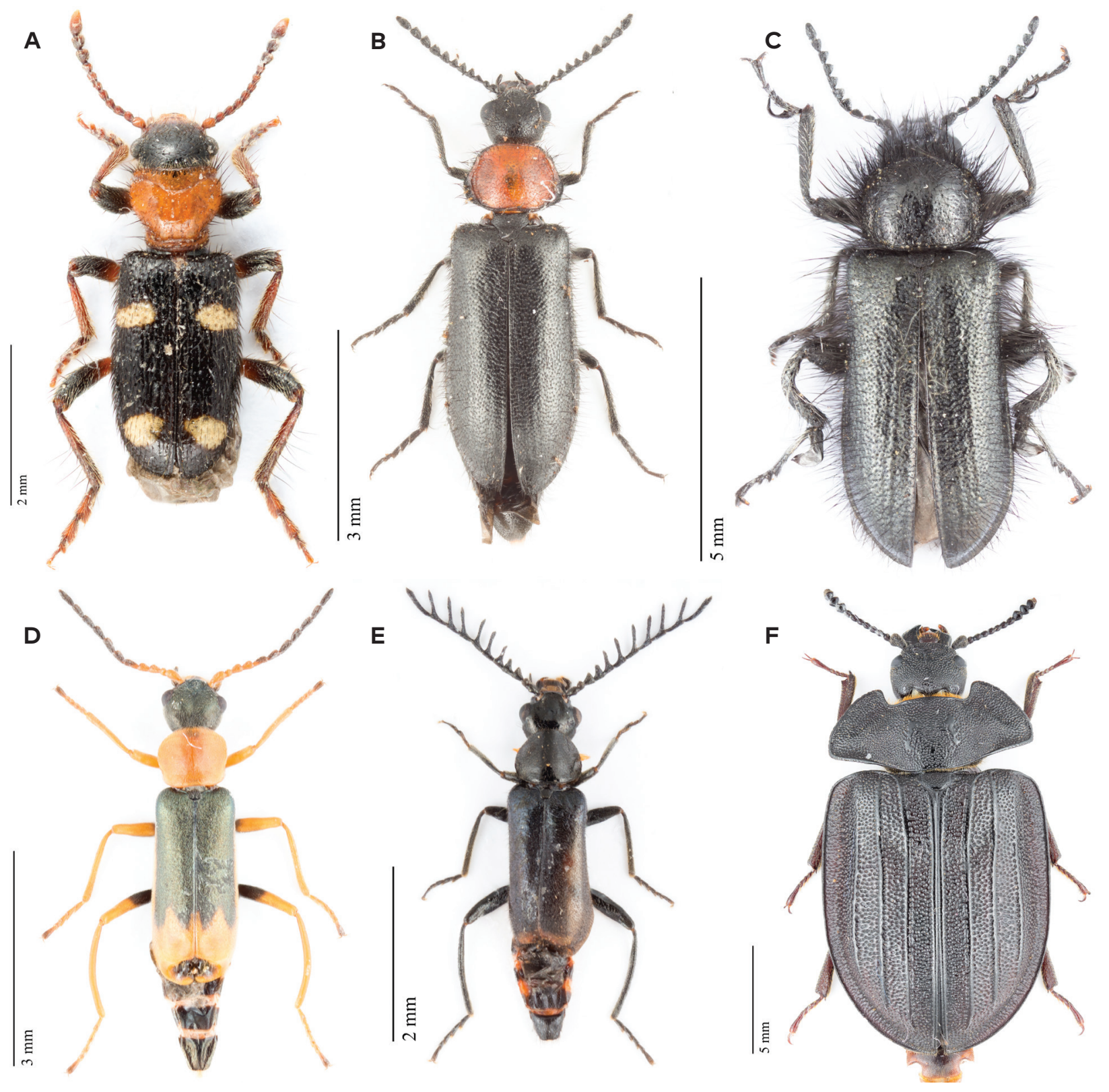

Figure 1. Habitus de quelques espèces de Cleroidea présentes en Suisse. A) Allonyx quadrimaculatus, B) Dasytes thoracicus, C) Enicopus pilosus, D) Cerapheles terminatus, E) Nepachys cardiacae, F) Peltis grossa (Photos A. Sanchez).

Commentaire. Cette espèce est connue d'Asie et d'Europe de l'Est (Pologne, Roumanie, Slovaquie et Ukraine) mais aussi de citations très anciennes d'Autriche, d'Allemagne (Niehuis 2013) et de Suisse (Müller 1912). Elle a également été découverte en Slovénie en 1993 (Niehuis 2013). Ces mentions d'Europe centrale sont à voir comme des populations relictuelles (Niehuis 2013) plutôt que comme des importations. Le spécimen suisse cité dans la littérature (Müller 1912) n'ayant pas été retrouvé et l'identification ne pouvant donc être confirmée (bien que l'espèce soit a priori inconfondable), T. ircutensis n'est pas considéré comme suisse en fonction des éléments à disposition. Dans tous les cas, T. ircutensis n'a plus été signalé en Suisse au cours des cent dernières années.

\section{C8) Dermestoides sanguinicollis}

Matériel examiné. ${ }^{3,4,6} 1$ ex., Basel, leg. \& coll. Staehlin-Bischoff H., NMB; ${ }^{3} 1$ ex., Valais, leg. Anonymous, MHNG; 1 ex., Chiasso, T. [Tannino], 4.7.1928, coll. Fontana P., MSNL; 1 ex., Tessin, Intragna Umg., 11.6.1966, leg. \& coll. Gehrig J., NMB; 1 ex., Someo, 26.6.1999, leg. \& coll. Gilgen M. \& Kamber L.; 1 ex., Rheinau ZH, 13.5.2007, leg. \& coll. Frei A.; 1 ex., Rheinau ZH, 20.5.2007, leg. \& coll Frei A.; 1 ex., Terre di Pedemonte TI, 4.-17.6.2013, leg. \& coll. Chittaro Y.

Données publiées. ${ }^{3,4,6} 1$ ex., Basel par Staehlin-Bischoff H. et ${ }^{3}$ Sitten par Gautard V. (Stierlin et Gautard 1867); 1 ex., Chiasso, Tannino, 1928, par Fontana P. (Fontana 1929). 
Commentaire. Rare mais assez largement distribuée en Europe, cette espèce saproxylique n'est connue que de quelques données de Suisse, principalement du Tessin et de quelques localités du nord du pays. Elle se développe principalement dans les chênes (Quercus spp.) et le châtaignier (Castanea sativa), souvent dans des arbres colonisés par le Grand capricorne Cerambyx cerdo (Sanchez et al. 2018).

\section{C9) [Tarsostenus univittatus]}

Matériel examiné. ${ }^{3)} 1$ ex., Genève, coll. Maerky C., MHNG; ${ }^{3)} 1$ ex., Genf, coll. Linder A., ex coll. Täschler M., ETH; 1 ex., Peney, 12.7.1886, coll. Tournier H., MHNG; 1 ex., Kt. Tessin, Chiasso, 7.1928, leg. \& coll. Linder A., ETH; 1 ex., Basel, 8.1935, leg. \& coll. Handschin E., NMB; 17 ex., Genève, 17.4.1984, leg. Besuchet C., MHNG; 16 ex., Genève, Eaux-Vives, 5.1984, leg. Besuchet C., MHNG; 2 ex., Genève, Port-Franc, 7.1995, leg. Besuchet C., MHNG; 1 ex., Dübendorf ZH, 3.2016, leg. Schmidt M., NMSO; 1 ex., Sion VS, 2.-18.8.2017, leg. \& coll. Chittaro M.-C.

Données publiées. ${ }^{1)}$ Genf par Tournier H. (Stierlin et Gautard 1867); Chiasso, 28.6.1922 par Fontana P. (Fontana 1925); Chiasso, Tannino, 1928 par Fontana P. (Fontana 1929).

Commentaire. Originaire probablement d'Australie, cette espèce est maintenant cosmopolite. En Suisse, elle a toujours été trouvée isolément et généralement en lien à des importations de bois (dans une tannerie au sud $\mathrm{du}$ Tessin, dans une menuiserie et un port-franc à Genève,...). La dernière capture, à Sion en 2017, a néanmoins été réalisée in natura. Les données disponibles actuellement ne permettent pas de savoir si l'espèce est réellement capable de s'établir en Suisse, comme cela a été le cas localement dans le sud de l'Allemagne (Geis 2001). De ce fait, elle est pour l'heure considérée comme ne faisant pas partie de la faune suisse.

\section{C10) Denops albofasciatus}

Matériel examiné. 1 ex., Cavigliano TI, 17.-30.6.2013, leg. \& coll. Chittaro Y.

Données publiées. 1 ex., Cavigliano TI, 17.-30.6.2013 par Chittaro Y. (Chittaro 2014).

Commentaire. Présente dans une grande partie de l'Europe méridionale, cette espèce n'est pour l'heure connue en Suisse que d'un unique exemplaire tessinois, capturé au piège d'interception dans une châtaigneraie.

\section{C11) [Danacea (Allodanacaea) wittmeri]}

Matériel examiné. ${ }^{8} 2$ ex., Lago di Lugano, Castagnola, 25.5.1924, leg. Anonymous, det. Liberti G., coll. Wittmer W., NMB.

Commentaire. Cette espèce endémique de la région de Naples (Liberti 1985) n'est assurément pas présente en Suisse. Il s'agit donc d'une erreur d'étiquettage des spécimens du NMB ou d'une importation accidentelle.

\section{C12) [Danacea (Danacea) iners]}

Matériel examiné. ${ }^{3,8} 1$ ex., Genf [Genève], det. Liberti G., coll. Lasserre H., MHNG.

Commentaire. La donnée de «Genf» provient d'une collection problématique qui ne doit pas être considérée. Cette espèce balkanique, atteignant à peine la frontière italienne, n'est pas indigène en Suisse. Mentionnée par Mayor (2007a) sous D. serbica, elle a été récemment synonymisée avec $D$. iners (Liberti 2009a).

\section{C13) [Danacea (Danacea) marginata]}

Données publiées. ${ }^{1,8)}$ Sta. Maria, Costeras, 19.6.1953 par Handschin E. (Handschin 1963).

Commentaire. Cette espèce colonise la partie méridionale de l'Europe de l'est et de l'Italie mais est toutefois absente d'Autriche, ce qui ne soutient guère une présence dans l'est de notre pays. Allenspach et Wittmer (1979) mettaient déjà en doute l'annonce de Handschin (1963), aucun individu de référence n'étant présent dans sa collection. D. marginata est à considérer comme absente de Suisse.

\section{C14) Danacea (Danacea) montivaga}

Matériel examiné. 2 ex., Chur, coll. Killias E., NMB; 2 ex., Schweiz, leg. Anonymous, NMB; 1 ex., Wallis, leg. Anonymous, ETH; 1 ex., Loèche-les-Bains, 16.5.1880, coll. Bugnion E., det. Constantin R., MZL; 2 ex., Kt. Wallis, Saastal, Saas-Almagell, 8.1939, leg. \& coll. Lautner J., det. Liberti G., NMB; 1 ex., La Forcla [La Forclaz VS], 24.7.1951, leg. \& coll. Simonet J., det. Liberti G., MHNG; 3 ex., Tessin, Mte. Gambarogno, 23.7.1980, leg. \& coll. Scherler P., det. Liberti G., NMBE.

Données publiées. ${ }^{1)}$ Simplon? [avec indication de doute] par Rätzer A. et ${ }^{1)}$ Val Ferret? [avec indication de doute] par Rätzer A. (Rätzer 1888); ${ }^{1)}$ Chandolin par Favre E., ${ }^{1)}$ Forclaz par Tournier H., Loèche-les-bains par Bugnion E., ')Mauvoisin par Bugnion E. et ${ }^{1)}$ Saas par Benteli F. (Favre 1890).

Commentaire. Cette espèce alpine rare est endémique des Alpes occidentales de France (de Chamonix au Mercantour), d'Italie (des Alpes de Ligurie au Monte Rosa) et de Suisse (Constantin et Liberti 2011), d'où elle est attestée avec certitude (examen de l'édéage) de quelques individus mâles provenant du Valais, mais aussi du Tessin.

\section{C15) [Danacea (Danacea) morosa]}

Données publiées. ${ }^{1)}$ Sierre par Steck-Hofmann T. et ${ }^{1)}$ Visperterminen par Bugnion E. (Favre 1890); ${ }^{1)}$ Chiasso, 5. par Fontana P. (Fontana 1925).

Commentaire. Cette espèce d'Europe centrale (sud de la Pologne, République tchèque, Slovaquie, Autriche, Hongrie), présente marginalement dans les Balkans et dans le nord est de l'Italie (Liberti 2009a), n'est pas présente en Suisse. Aucun individu de référence soutenant les citations de la littérature n'existe en effet dans les collections muséales suisses. 


\section{C16) [Danacea (Danacea) nigripalpis]}

Matériel examiné. ${ }^{3,8} 4$ ex., Sierre, coll. Favre E., det. Liberti G., MHNG.

Commentaire. Cette espèce endémique de Sicile (Liberti 1989) n'appartient assurément pas à la faune de Suisse. Les individus étiquetés de Sierre sont donc vraisemblablement le fruit d'erreurs d'étiquetage.

\section{C17) Danacea (Danacea) nigritarsis s.l.}

Matériel examiné. Nombreux exemplaires, principalement du Valais et du sud des Alpes, mais aussi isolément au nord des Alpes et sur le Plateau.

Commentaire. Deux sous-espèces de cette espèce politypique sont représentées en Suisse (Constantin et Liberti 2011): la sous-espèce alpina, largement répandue sur le versant sud des Alpes, et la sous espèce nominale. Nous n'avons toutefois pas procédé à la dissection systématique des individus mâles de $D$. nigritarsis, si bien qu'il est difficile de fournir une répartition fine des deux sous-espèces.

\section{C18) Dasytes (Anthoxenus) subaeneus}

Matériel examiné. ${ }^{3)} 1$ ex., Nyon, coll. Maerky C., ex coll. Poncy E., det. Constantin R., MHNG; ${ }^{3)} 1$ ex., Alpes, Tessin, coll. Maerky C., ex coll. Ghidini A., MHNG; ${ }^{3)} 1$ ex., Bienne, 6.6., leg. \& coll. Maerky C., MHNG; 1 ex., Auenst. [Auenstein AG], 17.7., leg. Anonymous, MHNG; 1 ex., Chancy GE, 13.7.1987, leg. \& coll. Scherler P., NMBE; 1 ex., Genève, Chancy, La Laire, 13.7.1987, leg. Besuchet C., MHNG; 1 ex., Dittingen BL, 10.6.2017, leg. \& coll. Chittaro Y.

Données publiées. ${ }^{1)}$ Genf par Chevrier F., ${ }^{1)}$ Genf par Lasserre H., ${ }^{1)}$ Genf par Tournier H. et ${ }^{1)}$ Jura par Heer O. (Stierlin et Gautard 1867); ${ }^{1)}$ Chiasso, 5. par Fontana P. (Fontana 1925); ${ }^{2}$ Innufer, 25.5.1953, ${ }^{1}$ Las Ruinas, 18.6.1953, ${ }^{2)}$ Costeras, 19.6.1953, ${ }^{2}$ Scuol-Ramosch, 30.6.1953, ${ }^{1)}$ Zernez, 20.6.1955 et ${ }^{2}$ Sta. Maria, 23.6.1955 par Handschin E. (Handschin 1963); ${ }^{1)}$ Weissenburg-Simmental BE par Huguenin E., ${ }^{2)}$ Lac Bleu-Arolla-V. d'Hérens VS [par Besuchet C.], MHNG (Allenspach et Wittmer 1979).

Commentaire. Largement répandue en Europe, cette espèce est pourtant très rare en Suisse, où sa présence n'est attestée que par quelques spécimens provenant des cantons de Genève, de Bâle-Campagne et d'Argovie. La plupart des citations de la littérature sont invérifiables, mais celles qui ont pu être contrôlées se reportaient à d'autres espèces du genre Dasytes.

\section{C19) [Dasytes (Dasytes) cf. murinus]}

Matériel examiné. ${ }^{3,6,8)} 1$ ex., Suisse, Nyon, leg. Anonymous, det. Liberti G., coll. Maerky C., MHNG.

Commentaire. L'individu examiné se rapproche de $D$. murinus, une espèce connue uniquement de Grèce et de Turquie. Provenant d'une collection problématique, il ne doit pas être considéré. Cette espèce n'est pas présente en Suisse.

\section{C20) Dasytes (Dasytes) pauperculus}

Matériel examiné. 1 ex., Genève, Avully, 5.-6.1993, leg. Besuchet C., det. Majer K., MHNG; 1 ex., Neuchâtel NE, 9.-25.5.2010, [leg. Haenni J.-P.], MHNN; 1 ex., Maggia TI, 6.4.-10.5.2014, leg. \& coll. Chittaro Y.

Commentaire. Trois spécimens seulement attestent de la présence en Suisse de cette espèce connue également d'Espagne, de France, d'Italie et de Slovénie. Les individus du Tessin et de Neuchâtel, deux femelles, ont été capturés au moyen de pièges d'interception placés dans de vieilles chênaies, une situation déjà documentée auparavant (Liberti 2009b).

\section{C21) Dasytes (Dasytes) thoracicus thoracicus Fig. 1B}

Matériel examiné. 1 ex., Wallis, Gondo, 14.7.1954, leg. Wolf J. P., MHNG; 1 ex., Tessin, Bordei, 21.7.1981, leg. Besuchet C., MHNG; 1 ex., Anzonico TI, 10.6.1997, leg. \& coll. Scherler P., NMBE; 30 ex., Zwischbergen VS, 1.7.2018, leg. \& coll. Breitenmoser S., Chittaro M.-C. \& Chittaro Y.

Données publiées. Anzonico par Focarile A. (Liberti et Focarile 2005).

Commentaire. En Suisse, cette espèce relique à distribution discontinue n'était connue jusqu'à récemment que de trois spécimens capturés dans le nord ouest du Tessin et sur le versant sud du Simplon. En 2018, elle a pu être capturée en grand nombre à proximité de l'ancienne station valaisanne. Les individus se trouvaient dans un mélèzin clair, à $1350 \mathrm{~m}$ d'altitude, perchés au sommet de graminées (Festuca rubra aggr., det. Godat Saskia \& Eggenberg Stefan). C'est la sous-espèce nominale, dont les femelles ont le thorax de couleur rouge (Fig. 1B), qui est présente en Suisse, en France et en Italie dans les Alpes occidentales (Alpes de Ligurie et Val d'Aoste) et dans les Appenins septentrionaux (Liberti et Focarile 2005).

\section{C22) Dasytes (Hypodasytes) lombardus}

Matériel examiné. 1 ex., Chiasso, 5.7.1937, coll. Fontana P., MSNL; 2 ex., Camoghé TI, 8.1963, leg. Toumayeff G., det. Constantin R., MHNG; 1 ex., Tessin, Camoghé, 6.8.1963, leg. Besuchet C., det. Constantin R., MHNG; 6 ex., Tessin, Somazzo, 11.7.1969, leg. \& coll. Scherler P., NMBE; 2 ex., Mte. San Giorgio TI, 22.7.1977, leg. \& coll. Scherler P., NMBE.

Données publiée. Chiasso (Fontana 1947); ${ }^{2)} 1$ ex., Salorino, 6.1940 par Lautner J. (Allenspach et Wittmer 1979); Cima di Foiorina (Colla), Gazzirola (Colla), Monte Bar et Capriasca TI, 6.2004 par Focarile A. (Liberti et Focarile 2005).

Commentaire. Les trois espèces Dasytes alpigradus, $D$. gonocerus et $D$. lombardus sont morphologiquement 
très proches et seuls les mâles peuvent être identifiés de manière certaine, après dissection et examen de l'édéage. Elles occupent cependant des aires de répartition globalement distinctes en Suisse (Fig. 2), avec seulement quelques zones de contact connues, notamment au Tessin (Liberti et Focarile 2005). D. lombardus, endémique des Préalpes calcaires lombardes, de l'Adamello aux Alpes Orobie et aux montagnes qui entourent le lac de Côme (Liberti et Focarile 2005), est la plus localisée des trois et ne se rencontre en Suisse que dans l'est du Tessin et dans le Val Mesolcina, où ses populations sont dans la continuité de celles du nord de l'Italie.

\section{C23) [Dasytes (Hypodasytes) tristiculus]}

Matériel examiné. ${ }^{3,8)} 1$ ex., Alpes, Sierre, 6.7., coll. Maerky C., det. Majer K., MHNG; ${ }^{3,8} 1$ ex., Jura, Argovie, leg. Anonymous, coll. Maerky C., ex coll. Frey-Gessner E., det. Majer K., MHNG.

Commentaire. Connue du nord-est de l'Espagne, d'Italie tyrrhénienne (Constantin et Liberti 2011) et de la moitié méridionale de la France et de Corse, cette espèce n'est pas retenue pour la Suisse, les deux individus examinés provenant d'une collection problématique.

\section{C24) [Dasytes (Mesodasytes) aeneiventris]}

Données publiées. ${ }^{1,8)}$ Loèche-ville et ${ }^{1,8}$ Lausanne par Bugnion E. (Favre 1890).

Commentaire. Cette espèce méridionale est connue de France, de Grèce, d'Italie, d'Espagne, d'Algérie, du Maroc et de Tunisie, ce qui ne soutient guère les citations de la littérature suisse (Favre 1890), pour lesquelles il n'existe par ailleurs aucun individu de référence.

\section{C25) Dasytes (Metadasytes) nigrocyaneus}

Matériel examiné. 1 ex., Indemini TI, 14.6.1974, leg. \& coll. Spälti A., det. Liberti G., MHNG; 2 ex., Vaud, Bonmont, 5.-7.1991, leg. Besuchet C., det. Majer K., MHNG; 1 ex., Vaud, Genolier, 5.-7.1991, leg. Besuchet C., det. Majer K., MHNG.

Données publiées. ${ }^{2)} 1$ ex., Lausanne, 7 . par Toumayeff G. (Allenspach et Wittmer 1979).

Commentaire. Cette espèce très rare, bien que largement distribuée en Europe occidentale et centrale, est connue de quelques observations de France, d'Allemagne, d'Autriche, de Hongrie et de Slovaquie (Constantin 2014). En Suisse, elle n'est attestée que par trois données du canton de Vaud et du Tessin. Le spécimen de Lausanne de Toumayeff G., signalé dans la littérature (Allenspach et Wittmer 1979), était un D. aeratus.

\section{C26) [Dasytidius nigripes]}

Matériel examiné. ${ }^{3,8} 1$ ex., Suisse, Gilly, 6.6., coll. Maerky C., MHNG; ${ }^{3,8} 1$ ex., Suisse, Gilly, 8.6., coll. Maerky C., MHNG.
Commentaire. Les deux spécimens examinés sont issus d'une collection problématique qui ne doit pas être considérée. Cette espèce n'est connue que d'Azerbaidjan, de Grèce (Rhodes), de Chypre, d'Iran, d'Iraq, de Syrie et de Turquie et n'appartient pas à notre faune.

\section{C27) [Divales quadrimaculatus]}

Matériel examiné. ${ }^{3,8} 3$ ex., Bienne, leg. \& coll. Maerky C., MHNG; ${ }^{3,8} 3$ ex., Bienne, 18.6., leg. \& coll. Maerky C., MHNG.

Commentaire. Cette espèce est connue uniquement de Provence en France et, très marginalement, de la province italienne d'Imperia en Ligurie occidentale (Constantin et Liberti 2011). Les seuls spécimens «suisses» que nous ayons pu examiner proviennent d'une collection problématique qui ne doit pas être considérée. L'espèce ne fait pas partie de notre faune.

\section{C28) [Enicopus (Enicopus) ater ater]}

Matériel examiné. ${ }^{3)} 2$ ex., Valais, coll. Maerky C., ex coll. Fries A., MHNG.

Commentaire. Ce n'est que récemment que le statut taxonomique de cete espèce a été clarifié (Liberti et Constantin 2009). Il s'agit d'une espèce avec une large distribution, de l'Europe centrale jusqu'au Kirghizistan, qui est notamment connue du sud de la France et d'Italie (Constantin et Liberti 2011). Les seuls spécimens examinés provenant d'une collection problématique, l'espèce n'est pas retenue pour la Suisse.

\section{C29) Enicopus (Enicopus) pilosus (Fig. 1C)}

Matériel examiné. ${ }^{3)} 1$ ex., Alpes, Valais, leg. \& coll. Maerky C., MHNG; 1 ex., Wallis, Gr. St. Bernard, coll. Cerutti N., MHNG; 1 ex., Gabi-Simplon, 6.-8.1948, leg. Weber P., coll. Allenspach V., NMB; 30 ex., Simplon VS, 30.6.2018 \& 11.7.2018, leg. \& coll. Breitenmoser S., Chittaro M.-C, Chittaro Y. \& Monnerat C.; 3 ex., Simplon VS, 11.7.2018, leg. \& coll. Chittaro Y.

Données publiées. ${ }^{1)}$ Schaffhausen par Stierlin G. (Stierlin et Gautard 1867); ${ }^{1)}$ Aigle par Jaccard H., ${ }^{7}$ Versant sud du St-Bernard [Col du Grand Saint-Bernard] par Favre E. (Favre 1890); ${ }^{1)}$ Rocca Bella par Rühl F. (Caflisch 1894); ${ }^{1)}$ Zernez, Clüs, 7.1937 par Handschin E. (Handschin 1963); ${ }^{1,3} 3$ ex., Le Vaud-Begnins, [coll. Maerky C.], MHNG; ${ }^{1,3} 5$ ex., Tessin, [coll. Maerky C.], MHNG (Allenspach et Wittmer 1979).

Commentaire. Cette espèce est connue du versant espagnol des Pyrénées, du sud-est de la France, de la quasi-totalité de l'Italie continentale ainsi que de Sicile (Constantin et Liberti 2011). Jusqu'à récemment, elle n'était connue en Suisse que de deux spécimens valides, l'un capturé en 1948 à Gabi, au sud du Simplon, et l'autre au Grand-SaintBernard (capture sans indication de date mais antérieure à 1900). S'agissant de deux femelles, la détermination spécifique demeurait néanmoins incertaine. En 2018, plu- 


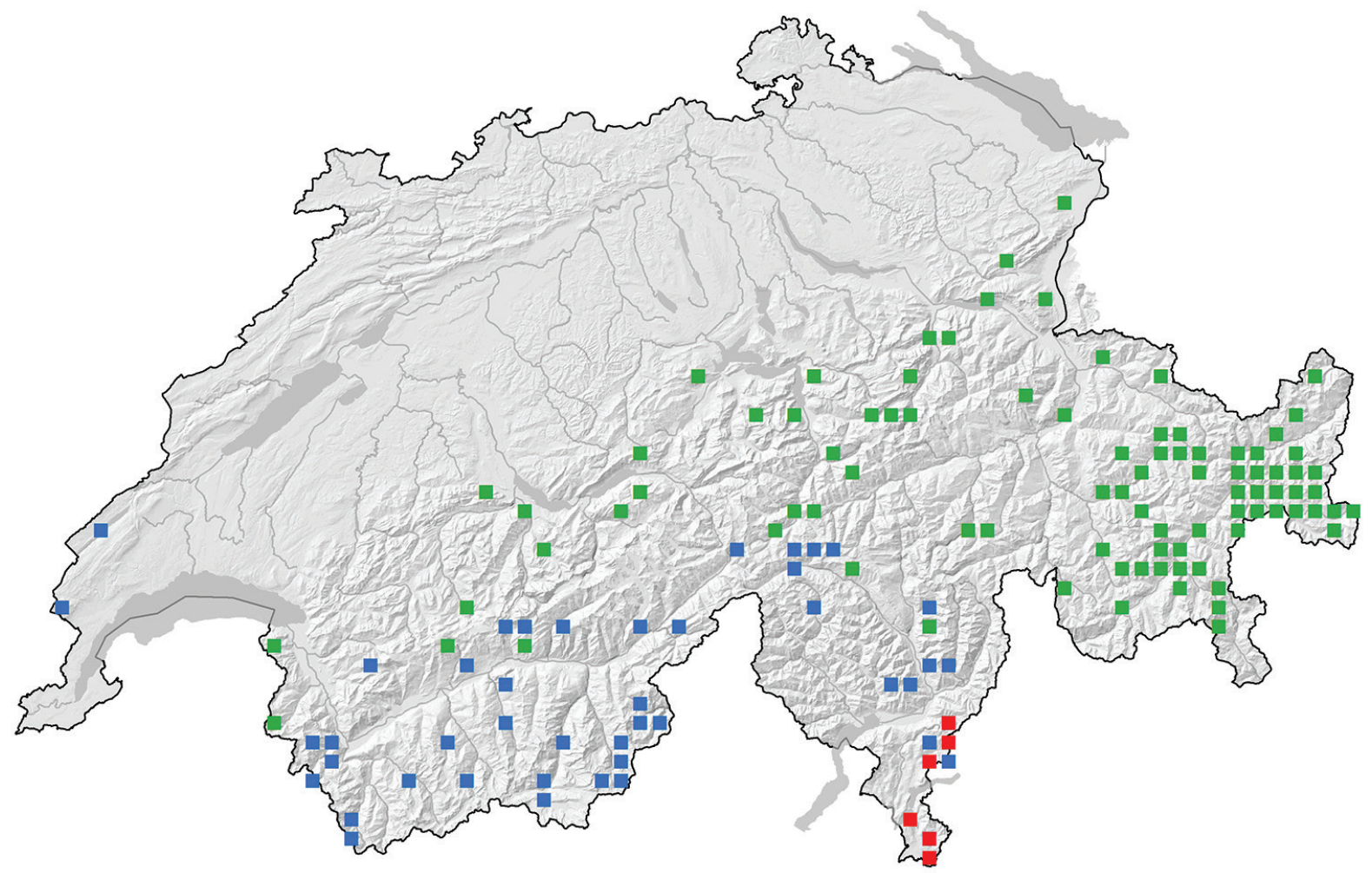

Figure 2. Répartition en Suisse de Dasytes alpigradus (en vert), D. gonocerus (en bleu) et D. lombardus (en rouge).

sieurs dizaines d'individus ont pu être observés, perchés le long de tiges de graminées, dans des pentes séchardes de mélèzins clairs à $1500 \mathrm{~m}$ d'altitude, à proximité de la localité historique du Simplon. Les spécimens mâles capturés permettent d'assurer l'identification spécifique et de confirmer l'existence d'une population sur territoire helvétique, dans la continuité de celles du nord de l'Italie, où elle est connue de diverses localités collinéennes et montagnardes. Elle semble par contre avoir disparu du Sud-Tyrol italien (Kahlen et Hellrigl 1996). Les autres citations suisses de la littérature sont invérifiables, ou, dans le cas des citations d'Allenspach et Wittmer (1979), concernent des spécimens femelles (en outre issues d'une collection problématique) qui ne peuvent être identifiés de manière certaine (Constantin et Liberti 2011).

\section{C30) [Psilothrix (Psilothrix) aureola]}

Données publiées. ${ }^{1,8)}$ Schaffhausen par Vogler (Stierlin 1883).

Commentaire. Cette espèce méditerrannéenne, connue des îles Baléares jusqu'au sud de la Grèce, ainsi que d'Afrique du Nord et des deux tiers méridionaux de l'Italie (Liberti 2009b), n'a été annoncée en Suisse que dans la littérature. Il n'existe pas d'individu de référence. $P$. aureola n'est dont pas retenu pour la Suisse.

\section{C31) [Psilothrix (Psilothrix) smaragdina]}

Données publiées. ${ }^{1)}$ Schaffhausen (Stierlin 1898).
Commentaire. Aucun matériel de référence suisse n'existe pour cette espèce, connue uniquement d'Italie et de Tunisie. Elle ne fait pas partie de notre faune.

\section{C32) Anthocomus (Celidus) humeralis}

Matériel examiné. 1 ex., Hasle-Rüegsau, 9.1972, leg. Kiener S., det. Švihla V., MHNG; 1 ex., Zürich, Rüti, 29.5.1973, leg. Stankowski B., det. Liberti G., MHNG.

Commentaire. En fonction des caractères donnés par Kolibáč et al. (2005), deux femelles capturées en Suisse peuvent être attribuées à cette espèce. Des spécimens supplémentaires seraient néanmoins bienvenus pour confirmer son indigénat. A. humeralis est cité d'Autriche, de Bulgarie, de République tchèque, de Grèce, de Roumanie, de Slovaquie, de Russie et de Turquie (Mayor 2007a).

\section{C33) [Anthomalachius spinosus]}

Matériel examiné. ${ }^{3)} 1$ ex., Bienne, coll. Maerky C., ex coll. Poncy E., det. Liberti G., MHNG; ${ }^{3)} 1$ ex., Nyon, coll. Maerky C., ex coll. Poncy E., det. Liberti G., MHNG; ${ }^{3)} 1$ ex., Zürich, coll. Maerky C., ex coll. Poncy E., det. Liberti G., MHNG.

Données publiées. ${ }^{1)}$ Dübendorf par Bremi-Wolf J.J., ${ }^{1)}$ Genf par Tournier H. et ${ }^{1)}$ Wallis par Stierlin G. (Stierlin et Gautard 1867); ${ }^{1}$ Martigny par Favre E. (Favre 1890); ${ }^{1)}$ Aigle par Jaccard H. (Jaccard 1890); ${ }^{1)}$ Martigny, MZL (Allenspach et Wittmer 1979). 
Commentaire. Largement répandue en Europe, cette espèce n'est pourtant pas retenue pour la Suisse en fonction des données à disposition. Les seuls spécimens «suisses» examinés proviennent en effet d'une collection qui ne doit pas être considérée, tandis que les citations issues de la littérature demeurent invérifiables.

\section{C34) [Attalus (Abrinus) amictus]}

Données publiées. $\left.{ }^{1,8}\right) 1$ ex., Wallis par Imhof L. (Stierlin et Gautard 1867); ${ }^{1,8}$ Siders et ${ }^{1,8)}$ Visp par Rätzer A. (Rätzer 1888); ${ }^{1,8}$ Martigny par Favre E. et ${ }^{1,8}$ Sierre par Böschenstein A. (Favre 1890); ${ }^{2,8}$ Nombreuses citations du canton du Valais (Allenspach et Wittmer 1979).

Commentaire. Annoncé de nombreuses localités valaisannes par Allenspach et Wittmer (1979), tous les individus que nous avons retrouvés et vérifiés sont à attribuer en réalité à l'espèce voisine $A$. analis. A. amictus, connu d'Espagne, du Portugal et du sud-ouest de la France (Constantin 2014), n'est donc pas considéré comme suisse en fonction des éléments disponibles.

\section{C35) Attalus (Attalus) alpinus}

Matériel examiné. 1 ex., Chandolin, coll. Favre E., MHNG; 1 ex., S-chanf, Godgod, [27.7.-4.]8.1981, leg. Reser-Rezbanyai L., coll. Wittmer W., NMB; 1 ex., All'Acqua, Val Bedretto TI, 12.6.2007, leg., det. \& coll. Liberti G.

Données publiées. ${ }^{1)} 1$ ex., Saas nach Fee, 1861 par Kiesenwetter H. (Kiesenwetter 1861); ${ }^{1)}$ Pontresina par Stierlin G. (Heyden 1863); ${ }^{1}$ Bevers et ${ }^{1}$ Rosegg, 6.1862 par Stierlin G. (Stierlin 1863); ${ }^{1)}$ Saas par Gautard V. (Stierlin et Gautard 1867); ${ }^{1)}$ Davos par Nagel H. (Caflisch 1894); Chandolin, ${ }^{1)}$ Ponchette et ${ }^{1)}$ Simplon par Favre E. (Favre 1890); 1 ex., S-chanf, Godgod, 27.7.-4.8.1981 par Reser-Rezbanyai L. (Herger 1994b).

Commentaire. Cette espèce très rare est présente uniquement dans les Alpes de France, d'Italie, d'Autriche et de Suisse. En Suisse, elle est confirmée uniquement de trois localités, du Valais, du Tessin et des Grisons, auxquelles viennnent s'ajouter quelques citations de littérature plausibles mais invérifiables. Le spécimen des Grisons (Godgod) a été capturé au piège lumineux alors que celui du Tessin (All'Acqua) a été obtenu au battage d'un épicéa (Picea abies) à $1520 \mathrm{~m}$ d'altitude.

\section{C36) [Attalus (Attalus) cyaneus]}

Matériel examiné. ${ }^{3,8)} 1$ ex., Genève, leg. \& coll. Poncy E., det. Švihla V., MHNG; ${ }^{3,8} 3$ ex., Sierre, coll. Bugnion E., det. Švihla V., MHNG.

Données publiées. ${ }^{1)}$ St. Bernhard (Stierlin 1898); ${ }^{1)}$ Chiasso (Fontana 1947).

Commentaire. Cette espèce méditerranéenne occidentale est connue du sud-est de la France (Alpes-Maritimes et Var selon Constantin 2014), d'Italie, de Malte, d'Espagne,
d'Algérie et de Tunisie. Nous ne la retenons pas pour la Suisse, les données publiées étant très anciennes et invérifiables, alors que les individus examinés sont issus de collections reconnues comme étant parfois problématiques.

\section{C37) Cerapheles terminatus}

Fig. 1D

Matériel examiné. 1 ex., Digue du Rhône [VD], 21.5.1918, coll. Gaud A., MZL; 19 ex., Kt. Waadt, Villeneuve, 5.1944, leg. Linder A., coll. Linder A. \& Allenspach V., ETH, NMB \& NMSO; 1 ex., Villeneuve, Grangettes, 24.5.1944, leg. von Peez A., coll. Pochon H., MHNF; 2 ex., Vaud, Seeufer Villeneuve, 27.5.1944, leg. von Peez A., MHNG; 2 ex., Kt. Waadt, Villeneuve, 6.1945, leg. \& coll. Linder A., ETH; 1 ex., Vaud, Neuveville [Villeneuve], 30.3.1946, leg. \& coll. Pochon H., MHNF; 1 ex., Kleinbösingen FR, 14.6.1991, leg. Hoess R., NMBE; 2 ex., Cudrefin VD, 11.7.1991, leg. \& coll. Scherler P., NMBE; 1 ex., Cudrefin VD, 5.6.1993, leg. \& coll. Scherler P., NMBE; 1 ex., Berne, Uebeschisee, 6.6.1996, leg. Besuchet C., MHNG; 2 ex., Cudrefin VD, 17.5.2018, leg. \& coll. Chittaro Y.; 1 ex., Avully GE, 24.5.2018, leg. \& coll. Chittaro Y.

Données publiées. Villeneuve, 5.1944 et 5.-6.1944 par von Peez A. et Villeneuve, 6.1945 par Linder A. (Linder 1946); ${ }^{11} 1$ ex., Les Echets-Caux, 5.1929, MHNG (Allenspach et Wittmer 1979); Portalban-Cudrefin, 11.6.1991 et 11.7.1991 par Scherler P. (Scherler 1995).

Commentaire. Cette espèce, assez largement répandue en Europe, colonise les roselières de bords de lacs et de grands cours d'eau. Elle n'est connue que de quelques localités de plaine en Suisse, toutes situées dans l'ouest du pays.

\section{C38) [Ceratistes dilaticornis]}

Données publiées. ${ }^{1}$ St. Gallen par Täschler M. (Stierlin 1883); ${ }^{1} 1$ ex., Bironico, 25.5.1901 par Fontana P. (Fontana 1925).

Commentaire. Aucun spécimen en collection ne vient soutenir les citations de la littérature. Cette espèce est principalement répandue dans le sud-est de l'Europe. Ses citations (anciennes) de France et d'Italie sont douteuses (Franzini G., comm. pers.).

\section{C39) Charopus docilis}

Matériel examiné. 1 ex., Kt. Tessin, Ascona, 6.1934, leg. \& coll. Linder A., ETH; 2 ex., Genève, Chancy, La Laire, 20.5.1985, leg. Besuchet C., det. Švihla V. \& Liberti G., MHNG.

Commentaire. Cette espèce méridionale est connue d'Italie, d'Espagne, d'Andorre, d'Algérie et de France. Dans ce dernier pays, elle remonte jusqu'en Bugey (Ain) notamment (Constantin 2014), ce qui soutient sa présence, seulement récemment mise en évidence, à Genève aussi. Un spécimen tessinois, très abîmé, a aussi été attribué à 


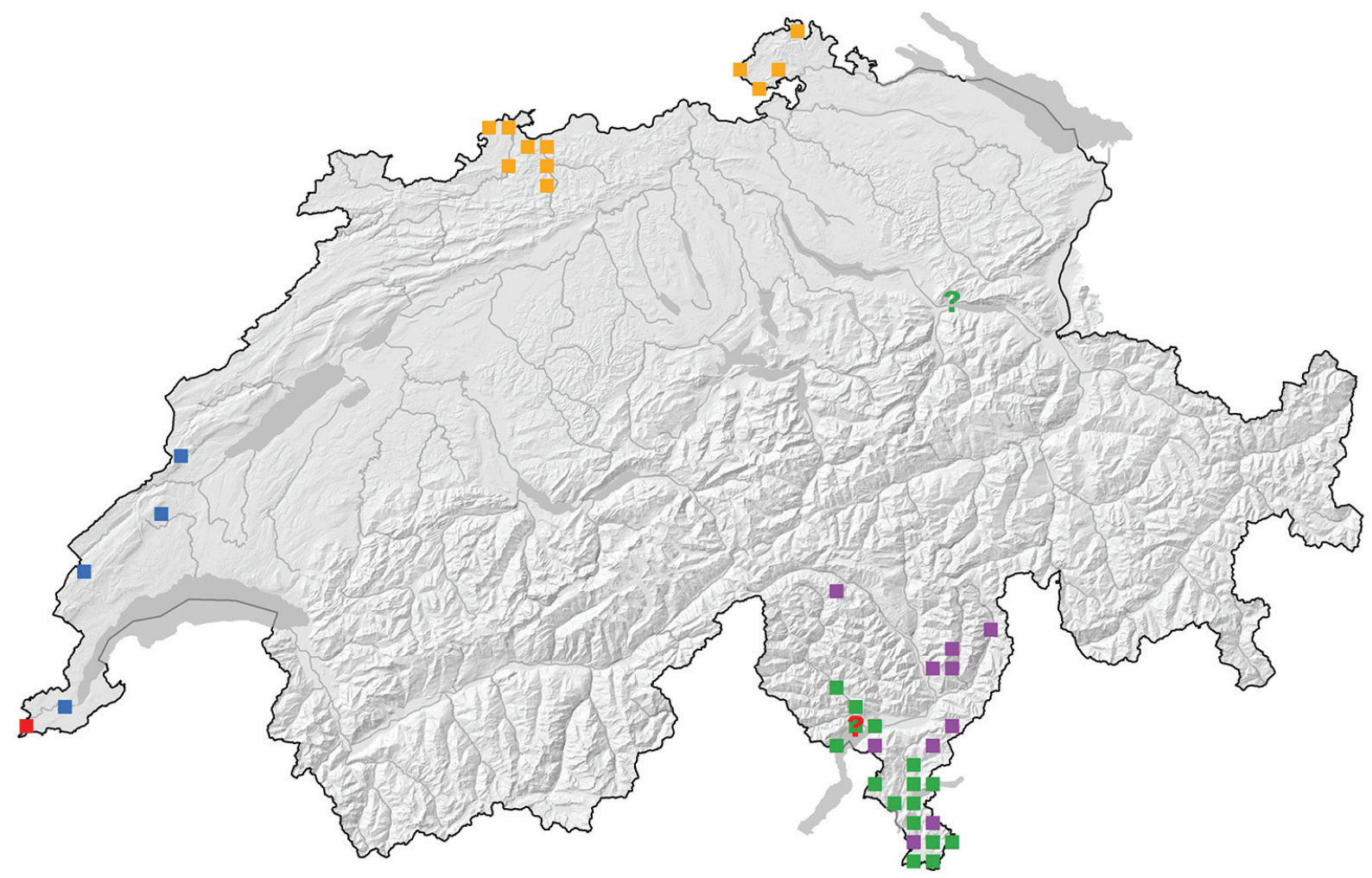

Figure 3. Répartition des espèces du genre Charopus en Suisse: C. concolor (en vert), C. docilis (en rouge), C. flavipes (en orange), C. madidus (en violet) et C. pallipes (en bleu). Une observation au nord des Alpes de C. concolor (1 ex., Betlis am Walensee, Gasthaus «Paradiesli», 4.7.1967, leg. Farmer R., NMB; aussi publiée par Allenspach (1968)) résulte probablement d'une importation et est signalée par un point d'interrogation vert. Une observation de C. docilis au Tessin est également incertaine, l'individu étant très abîmé (voir C39), et est indiquée par un point d'interrogation rouge.

cette espèce, mais une confusion avec $C$. concolor, commun dans la région (Fig. 3), n'est pas totalement exclue.

\section{C40) Charopus flavipes}

Matériel examiné. 1 ex., Löhningen, 2.6.1944, leg. \& coll. Allenspach V., NMB; 2 ex., Basler Jura, Bubendf. [Bubendorf], 30.6.1946, leg. Wolf J.P., ETH; 2 ex., Basler Jura, Ziefen, 30.6.1946, leg. Wolf J.P., ETH; 1 ex., Basler Jura, Tiefental, 12.6.1947, leg. Wolf J.P., ETH; 2 ex., Merishausen, 23.5.1948, leg. \& coll. Allenspach V., NMB; 1 ex., Muttenz BL, 5.1954, leg. Toumayeff G., MHNG; 2 ex., Bâle, Allschwil, 6.1955, leg. Toumayeff G., MHNG; 1 ex., Muttenz BL, 6.1955, leg. Toumayeff G., MHNG; 2 ex., Aesch BL, 7.1958, leg. Toumayeff G., MHNG; 1 ex., Kt. Basel, Augst, 6.1967, leg. \& coll. Linder A., ETH; 1 ex., Kt. Bern, Duggingen, 6.1967, leg. \& coll. Linder A., ETH; 1 ex., Allschwil BL, Ziegelei, 17.5.1978, leg. Kiener S., MHNG; 1 ex., Kt. BL, Allschwil, Ziegelei, 8.7.1978, leg. Kiener S., det. Śvihla V., MHNG; 1 ex., s/Hallau, Röti SH, 5.1984, leg. Toumayeff G., MHNG; 1 ex., Schaffhouse, s/Osterfingen, 9.6.1988, leg. Besuchet C., MHNG; 1 ex., Schaffhouse, Wangental près Osterfingen, 10.6.1988, leg. Besuchet C., MHNG; 2 ex., Bubendorf BL, 18.6.2005, leg., det. \& coll. Geiser M., NMBE; 1 ex., Basel BS, 16.18.6.2017, leg. \& det. Germann C., NMSO.
Données publiées. ${ }^{1)}$ Valais par Stierlin G. (Favre 1890); ${ }^{2}$ Chiasso, 14.6, par Fontana P. (Fontana 1925); ${ }^{2)}$ Generoso par Fontana P. (Fontana 1947); ${ }^{1)} 5$ ex., Noville, 5.1901, MZL, ${ }^{2)} 1$ ex., Mendrisio, 7.1940 par Allenspach V., ${ }^{2}$ Mte Generoso par Linder A., ${ }^{2)} 1$ ex., Mesocco, 7.-8.1943 par Allenspach V., ${ }^{2)} 1$ ex., Mte Generoso, 7.1964 par Scherler P., ${ }^{2)} 2$ ex., Mte Generoso, 8.1965 par Scherler P., ${ }^{2)} 2$ ex., Mte Generoso, 8.1967 par Scherler P., ${ }^{2)} 1$ ex., Mte Bisbino-V. Muggio-Italien, 7.1954, [leg. Besuchet C.], MZL, ${ }^{2)} 2$ ex., Tesserete-V. Colla, 7.1964, par Scherler P. et ${ }^{2)} 1$ ex., V. Calanca, 6.1948, par Allenspach V. (Allenspach et Wittmer 1979); Zoo Basel, secteur H (Sprecher et al. 2008).

Commentaire. Cette espèce est très largement répandue en Europe. En Suisse, elle se limite uniquement au nord du pays, contrairement à l'espèce voisine $C$. pallipes qui colonise plutôt le sud-ouest (Fig. 3). Les données publiées du Tessin sont à reporter en quasi-totalité à C. madidus.

\section{C41) Charopus madidus}

Matériel examiné. 2 ex., Chiasso, coll. Fontana P., MHNG; 1 ex., Generoso, 2.7.1926., coll. Fontana P., MSNL; 1 ex., Mendrisio TI, 10.-24.7.1940, leg. \& coll. Allenspach V., NMB; 1 ex., Mesocco GR, 6.-7.1943, leg. \& coll. Allenspach V., NMB; 1 ex., V. Calanca, 5.6.1948, 
leg. \& coll. Allenspach V., NMB; 1 ex., Kt. Tessin, Generoso, 7.1954, leg. \& coll. Linder A., ETH; 1 ex., Tessin, Mte. Generoso, 26.7.1954, leg. Besuchet C., det. Švihla V., MZL; 1 ex., Tessin, Mte. Bisbino, 27.7.1954, leg. Besuchet C., MZL; 1 ex., B. Vista [Bellavista, Monte Generoso], 12.7.1960, leg. Zuber A., det. Švihla V., MHNG; 1 ex., Camoghe TI, 8.1963, leg. Toumayeff G., MHNG; 1 ex., Tessin, Generoso, 14.7.1964, leg. \& coll. Scherler P., NMBE; 4 ex., Tessin, s/Colla, 20.7.1964, leg. \& coll. Scherler P., NMBE; 2 ex., Tessin, Generoso, 22.8.1965, leg. \& coll. Scherler P., NMBE; 1 ex., Tessin, Generoso, 14.8.1967, leg. \& coll. Scherler P., NMBE; 1 ex., Indemini, 7.1969, leg. Toumayeff G., MHNG; 1 ex., Tessin, Mt. Generoso, 22.7.1977, leg. Marggi W., MHNG; 5 ex., Tessin, Bella Vista, 11.7.1981, leg. Besuchet C., MHNG; 1 ex., Tessin, Somazzo, 15.6.1990, leg. \& coll. Scherler P., NMBE; 10 ex., Monte Generoso TI, Vetta, 13.7.1990, leg. Reser-Rezbanyai L., NMLU; 1 ex., Tessin, Alpe Brogoldone, s/Claro, 17.7.1990, leg. Besuchet C., MHNG; 2 ex., Molinera TI, 19.7.1990, leg. \& coll. Scherler P., NMBE.

Données publiées. Generoso (Fontana 1947); Capriasca TI, 6.2004 par Focarile A. (Liberti et Focarile 2005).

Commentaire. Plusieurs spécimens de diverses localités tessinoises (Fig. 3) confirment la présence en Suisse de cette espèce localisée, connue également d'Autriche, d'Italie, de Slovénie et des Balkans.

\section{C42) Charopus pallipes}

Matériel examiné. 1 ex., Vaud, Marchairuz, Roch, 27.6.1976, leg. \& coll. Scherler P., NMBE; 1 ex., Vaud, Moiry, 30.5.1982, leg. \& coll. Scherler P., NMBE; 1 ex., Genève, P1. des Nations, 28.6.1987, leg. Steffen J., MHNG; 1 ex., Vaud, Aig. [Aiguilles] de Baulmes, 3.8.1987, leg. \& coll. Scherler P., NMBE; 1 ex., Genève, Pl. des Nations, 7.7.1992, leg. Steffen J., det. Švihla V., MHNG.

Données publiées. ${ }^{1)}$ Basel par Bischoff-Ehinger A., ${ }^{1)}$ Genf par Stierlin G., ${ }^{1}$ Genf par Tournier H. et ${ }^{1)}$ Schaffhausen par Stierlin G. (Stierlin et Gautard 1867); 1)Poschiavo par Jörger J. (Linder 1967).

Commentaire. Moins largement répandue en Europe que $C$. flavipes, cette espèce est retenue pour la Suisse sur la base de quelques exemplaires mâles provenant de trois localités du canton de Vaud et d'une de Genève (Fig. 3).

\section{C43) Clanoptilus (Clanoptilus) affinis}

Matériel examiné. 1 ex., St. Maria, Costeras, 25.5.1953, leg. \& coll. Handschin E., BNM; 1 ex., St. Maria, Costeras, 19.6.1953, leg. \& coll. Handschin E., BNM; 1 ex., St. Maria, Valpaschun, 20.6.1953, leg. \& coll. Handschin E., BNM; 1 ex., Münstertal ob Müstair, 1.8.1956, leg. Eglin W., BNM; 2 ex., Müstair GR, 28.7.1970, leg. \& coll. Scherler P., det. Wittmer W., NMBE.

Données publiées. ${ }^{1)}$ Lägern par Isenschmid M., ${ }^{1)}$ Locarno par Isenschmid M. et ${ }^{1)}$ Siders par Isenschmid M. (Stierlin 1883); ${ }^{1)}$ Sierre et environs par Schacht W. (Favre 1890); ${ }^{1)}$ Maienfeld par Müller J.A. (Müller 1912); ${ }^{1)} \mathrm{Ge-}$ neroso (Fontana 1947); ${ }^{1)}$ Scuol, Gurlaina, 27.5.1953, Sta. Maria, Costeras, 25.5.1953 et Valpaschun, 20.6.1953 par Handschin E. (Handschin 1963); ${ }^{2}$ Les Grangettes-Villeneuve VD, ${ }^{2}$ Lötschental et ${ }^{2)}$ Sion par Pochon H., ${ }^{2}$ Morcote, MHNG, ${ }^{2)}$ Alpe di Neggia [par Besuchet C.], ${ }^{2)}$ Monti di San Abbondio-Ranzo [par Besuchet C.], MHNG, ${ }^{2}$ Locarno [par Gaud A.], MZL, ${ }^{2}$ Dandrio-V. Pontirone par Allenspach V., ${ }^{1)}$ Altanca-Ambri-Piotta, MZL, ${ }^{1)} 1$ ex., Siloah-Muri BE, 7.1953, MHNG, ${ }^{2} 1$ ex., Lutry VD, 5.1955, [par Aubert J.], MZL (Allenspach et Wittmer 1979).

Commentaire. Seuls six spécimens, tous capturés dans l'extrême est de la Suisse (Val Müstair, Fig. 4), permettent de considérer cette espèce comme faisant partie de la faune suisse. Tous les autres individus figurant sous "Clanoptilus affinis" dans les collections suisses examinées, pour certains cités par Allenspach et Wittmer (1979), appartenaient en réalité à d'autres espèces des genres Clanoptilus, Malachius ou Cordylepherus. A proximité des populations suisses, C. affinis est également connu du Vorarlberg (Brandstetter et Kapp 1998) et du Sud-Tyrol italien (Kahlen et Hellrigl 1996). Présent localement en France (Constantin 2014), C. affinis pourrait aussi se trouver dans l'ouest de la Suisse dans des zones xérothermophiles.

\section{C44) [Clanoptilus (Clanoptilus) arnaizi]}

Données publiées. ${ }^{1)}$ Suisse (Constantin 2014).

Commentaire. Nous n'avons pas connaissance de spécimens suisses se référant à cette espèce colonisant l'Italie, l'Espagne et la moitié méridionale de la France. Néanmoins, un spécimen du Val d'Aoste voisin (Italie), où l'espèce est commune, est conservé au MHNG. C'est peut-être de cet individu que découle la mention bibliographique. Pour l'heure, l'espèce n'est pas retenue pour la Suisse bien qu'elle y soit potentielle, notamment dans certaines zones xériques du Valais.

\section{C45) Clanoptilus (Clanoptilus) geniculatus}

Matériel examiné. ${ }^{3)} 1$ ex., Tarasp, leg. Anonymous, BNM; 3) 1 ex., Trins, leg. Anonymous, BNM; 1 ex., Bellinzona, 7.1914, coll. Kehrmann F., det. Švihla V., MHNG; 1 ex., Locarno TI, 5.1934, leg. Toumayeff G., MHNG; 1 ex., Chiasso, 26.5.1939, coll. Fontana P., MSNL; 8 ex., Kt. Graubünden, Grono, 6.1951, leg. \& coll. Linder A., ETH; 1 ex., Golino TI, 13.6.1987, leg. \& coll. Scherler P., NMBE.

Données publiées. ${ }^{1)}$ Menaggio par Meyer-Dür R. (Stierlin 1863-1864); ${ }^{1)}$ Genf par Chevrier F., ${ }^{1)}$ Genf par Tournier H. et ${ }^{1)}$ Schaffhausen par Stierlin G. (Stierlin et Gautard 1867); ${ }^{1)}$ Sierre et environs par Schacht W. (Schacht 1879); ${ }^{1)}$ Entremont, ${ }^{1)}$ Ferret, ${ }^{1)}$ Gadmen et ${ }^{1)}$ Pieterlen a. Jura par Rätzer A. (Rätzer 1888); ${ }^{1)}$ Domleschg par Stoffel A., ${ }^{1)}$ Schuls, ${ }^{1)}$ Tarasp et ${ }^{1)}$ Nairs, 1874 par Killias E., ${ }^{1)}$ Bergell par Bazzigher L. (Caflisch 1894); ${ }^{1)}$ Martigny, ${ }^{1)}$ Fully, ${ }^{1)}$ Isérables, ${ }^{1}$ Sierre et ${ }^{1)}$ Chandolin par Favre (Favre 1890), ${ }^{1)}$ Chiasso (Fontana 1925); ${ }^{1}$ Airolo (Fontana 1947); Grono par Linder A. (Linder 1967); ${ }^{2}$ Bölchen SO/ 
BL par Lautner J. \& Wittmer W; ${ }^{2}$ Lägern ZH par Lautner J.; ${ }^{1)}$ Biel BE; ${ }^{2,3)}$ Gilly [ex coll Maerky C.,MHNG]; ${ }^{2)} \mathrm{Ve}-$ vey [par Gaud A.], MZL; ${ }^{2}$ Pomy-Yverdon VD [par Mellet P., MZL]; ${ }^{2,3}$ Fribourg, [ex coll Maerky C.], MHNG, ${ }^{2)}$ Pérolles par Pochon H.; ${ }^{1)}$ Schwarzenburg BE par Toumayeff G., MHNG; ${ }^{2,3}$ Burgdorf BE, MHNG; ${ }^{1}$ Zürich par Huguenin H., ${ }^{2)}$ Muttenz BL par Toumayeff G., [MHNG]; ${ }^{2)}$ Baden AG par Toumayeff G., [MHNG]; ${ }^{2)}$ Bülach ZH par Lautner J. [NMB]; ${ }^{1)}$ V. Eau Froide par Scherler P.; ${ }^{2)}$ Roche par Scherler P., [NMBE]; ${ }^{2}$ Aigle VD par Scherler P., [NMBE]; ${ }^{2,3}$ Les Ormonts VD [coll Maerky C.], MHNG; ${ }^{1)}$ Gryon VD, MZL; ${ }^{2}$ La Comballaz par Toumayeff G., [MHNG]; ${ }^{2}$ Schweinsberg FR par Pochon H. [MHNF]; ${ }^{1)}$ Simmental BE par Huguenin H., MHNG; ${ }^{1)}$ Gadmen-Innertkirchen BE par Allenspach V.; ${ }^{1)}$ Baltschiedertal par Toumayeff G.; ${ }^{2)}$ Isérables [par Simonet J.], MHNG; ${ }^{1)}$ Vissoie, St. Luc par Spälti A.; ${ }^{2)}$ Nanztal-Brig par Toumayeff G., MHNG; ${ }^{1)}$ Scuol, MHNG; ${ }^{2)}$ Montagnola-Lugano par Kutter H., [MZL]; ${ }^{2}$ Sopraceneri, Cartada-Locarno par Brugger A. [MHNG] (Allenspach et Wittmer 1979).

Commentaire. Seuls quelques rares spécimens provenant du sud des Alpes (Fig. 4) permettent de considérer cette espèce, largement répandue à l'échelle paléarctique, comme suisse. Citée d'Alsace (Callot 2018), sa présence dans le nord du pays serait également envisageable. La plupart de spécimens cités par Allenspach et Wittmer (1979) qui ont été retrouvés appartenaient en réalité à d'autres espèces des genres Clanoptilus, Malachius ou Cordylepherus.

\section{C46) [Clanoptilus (Clanoptilus) rufus]}

Matériel examiné. ${ }^{4,8} 1$ ex., Lausanne, 7. , leg. Toumayeff G., MHNG.

Données publiées. ${ }^{4,8} 1$ ex., Lausanne, 7.7, par Toumayeff G., MHNG (Allenspach et Wittmer 1979).

Commentaire. Cette espèce méditerranéenne, connue d'Espagne, du Maghreb, d'Italie et de France, ne peut pas être retenue pour la faune de Suisse sur la base de l'unique exemplaire examiné. Bien qu'appartenant à une collection de qualité, l'étiquette de la localité laisse penser à une erreur d'étiquetage ou à un échange, Toumayeff G. mentionnant toujours l'année de découverte, ce qui n'est pas le cas ici.

\section{C47) [Clanoptilus (Clanoptilus) spinipennis]}

Données publiées. ${ }^{1,8)}$ Sierre et environs par Redtenbacher L. (Schacht 1879); ${ }^{1,8)}$ Chandolin, ${ }^{1,8}$ Niouc et ${ }^{1,8}$ Sierre par Favre E. (Favre 1890); ${ }^{1}$ Trins, 1890 par Caflisch J. (Caflisch 1894); ${ }^{2} 1$ ex., Bex VD [par Gaud A.], MZL, ${ }^{2}$ Pfynwald par Pochon H. [MHNF], ${ }^{1)}$ Mörel, MZL; ${ }^{2)}$ V. d'Anniviers, Niouc [par Gaud A.], MZL, ${ }^{1)}$ Bigorio-Tesserete et ${ }^{2)}$ Monti-Locarno par Spälti A. (Allenspach et Wittmer 1979).

Commentaire. Citée dans plusieurs références bibliographiques, aucun spécimen valide de cette espèce n'a cependant été trouvé dans les collections suisses examinées. La plupart de spécimens cités par Allenspach et Wittmer (1979) qui ont été retrouvés appartenaient en effet à d'autres espèces des genres Clanoptilus, Malachius ou Cordylepherus. Cette espèce est principalement répandue en Europe de l'est, jusqu'au Caucase et l'ouest de l'Asie. En Italie du Nord, elle n'est connue que de la côte adriatique (Franzini G., comm. pers.). Sa présence en Suisse est très peu probable.

\section{C48) [Clanoptilus (Hypoptilus) barnevillei]}

Données publiées. ${ }^{1)}$ Brigue par van der Hoop D. (Favre 1890); ${ }^{2} 2$ ex., Müstair, 7.1970 par Scherler P. (Allenspach et Wittmer 1979).

Commentaire. Cette espèce est connue localement de France, d'Italie, d'Espagne, d'Allemagne, d'Angleterre et de Suède. Citée de l'Ain et du Jura en France (Constantin 2014), une présence dans l'ouest de notre pays est possible, mais aucun spécimen suisse ne vient pour l'heure confirmer cette hypothèse. Les spécimens cités par Allenspach et Wittmer (1979) étaient des C. affinis, alors que le spécimen de Brigue n'a pas été retrouvé dans les collections consultées.

\section{C49) Cyrtosus (Oogynes) ovalis}

Matériel examiné. 1 ex., Caneggio, coll. Fontana P., MSNL; 5 ex., Chiasso, coll. Fontana P., MSNL; ${ }^{8)} 1$ ex., Valais, Euseigne, 6.6., leg. \& coll. Pochon H., MHNF; 3 ex., Mendrisio, 25.5.1930, leg. \& coll. Allenspach V., NMB; 1 ex., Chiasso, 6.6.1933, coll. Fontana P., MSNL; 1 ex., Mendrisio, 30.5.-2.6.1936, leg. \& coll. Lautner J., NMB; 14 ex., Kt. Tessin, Mendrisio, 5.1943, leg. \& coll. Linder A., ETH; 1 ex., Tessin, Mendrisio, 1.6.1943, leg. \& coll. Pochon H., MHNF; 2 ex., Mendrisio, 6.1949, leg. \& coll. Linder A., ETH; 2 ex., Chiasso, 6.1953, leg. Linder A., coll. Toumayeff G. \& coll. Lautner J., MHNG \& NMB; 1 ex., Kt. Tessin, Origlio, 6.1975, leg. Linder A., NMBE; 8 ex., Muzzano TI, 12.6.1985, leg. \& coll. Scherler P., NMBE; 5 ex., Muzzano TI, 20.6.1985, leg. \& coll. Scherler P., NMBE; 2 ex., Tessin, Lago di Muzzano, 24.6.1987, leg. Besuchet C., MHNG; 6 ex., Muzzano TI, 24.6.1987, leg. \& coll. Scherler P., NMBE.

Données publiées. Mendrisio, 5.1943 par Linder A. (Linder 1946); Chiasso (Fontana 1947).

Commentaire. En Suisse, cette espèce est connue de quelques localités tessinoises, dans la continuité de ses observations au nord de l'Italie. On la connaît aussi d'Espagne, de Grèce et de Turquie. La localité valaisanne est douteuse et résulte probablement d'une erreur d'étiquetage.

\section{C50) [Ebaeus (Ebaeus) appendiculatus]}

Données publiées. ${ }^{1)}$ Burgdorf par Meyer, ${ }^{1)} \mathrm{Genf}$ par Tournier H. et ${ }^{1)}$ Schaffhausen par Stierlin G. (Stierlin et Gautard 1867); ${ }^{1)}$ Siders par Stierlin G. (Stierlin 1883); ${ }^{1)}$ Nairs par Killias E. (Caflisch 1894); ${ }^{2} 1$ ex., Agiez-Orbe VD, 7.1949, [par Besuchet C., MZL], ${ }^{2)} 1$ ex., Veyrier GE, 7.1953, [par Simonet J., MHNG], ${ }^{1)} 1$ ex., Irchel-Winterthur, 


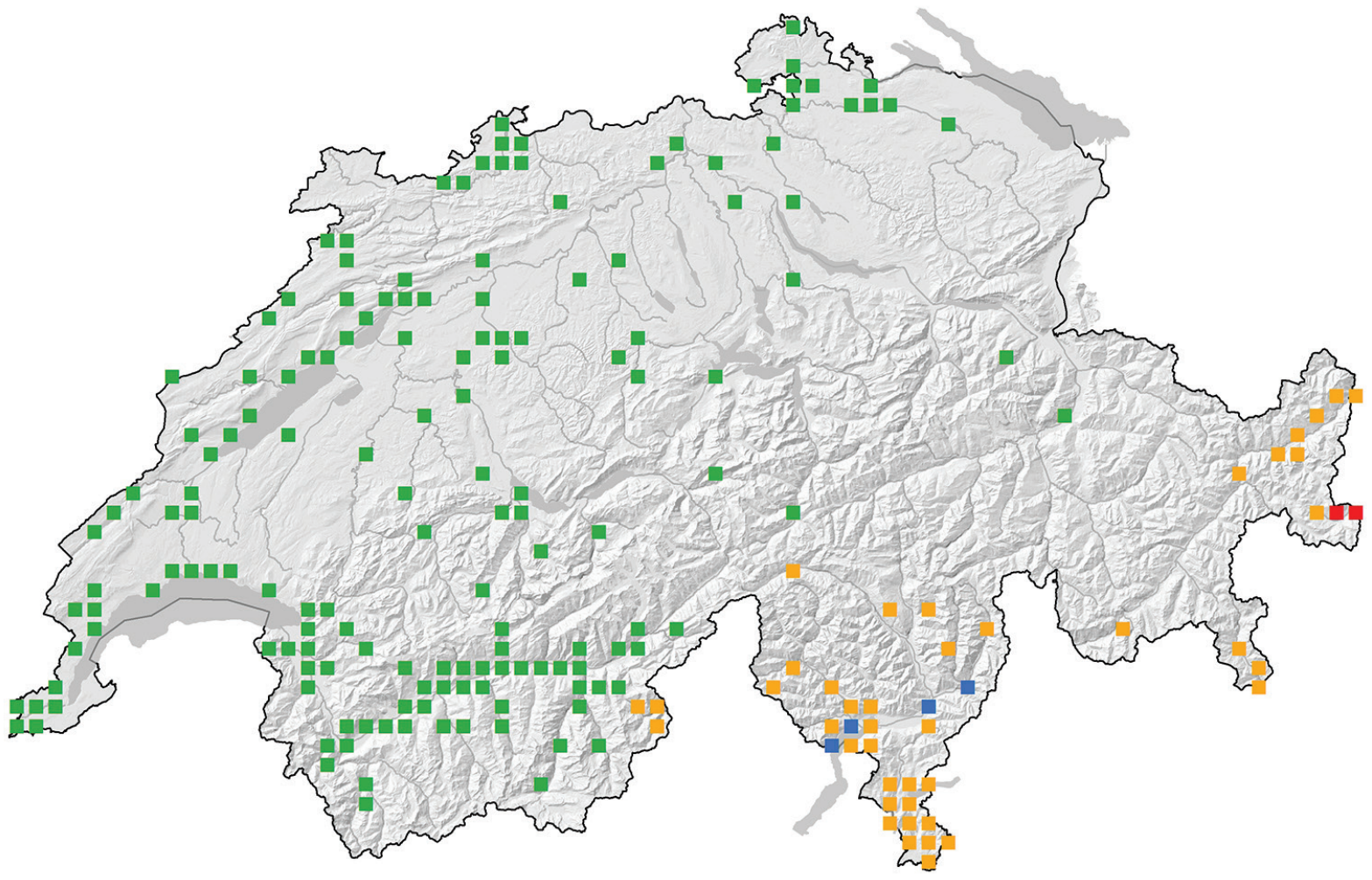

Figure 4. Répartition des espèces du genre Clanoptilus en Suisse, à l'exception de C. marginellus : C. affinis (en rouge), C. elegans (en vert), C. emarginatus (en orange) et C. geniculatus (en bleu). Les deux espèces sœurs C. elegans et C. emarginatus ne cohabitent pas.

NMB,${ }^{1)} 1$ ex., St-Triphon-Ollon, 5.1951, MZL,${ }^{2)} 1$ ex., Follaterres-Martigny, 6.1954 (Allenspach et Wittmer 1979).

Commentaire. Cette espèce colonise principalement les pays d'Europe centrale et de l'est. Citée plusieurs fois dans la littérature suisse, nous n'avons toutefois pas vu de spécimen de référence dans les collections examinées. Les quelques individus retrouvés se rapportaient en effet à d'autres espèces des genres Ebaeus ou Hypebaeus. Citée notamment du Sud-Tyrol italien (Kahlen et Hellrigl 1996) et du sud-est de l'Allemagne (Köhler et Klausnitzer 1998), sa présence est possible en Suisse, mais demande confirmation.

\section{C51) [Ebaeus (Ebaeus) ater]}

Données publiées. ${ }^{2} 1$ ex., Versoix, 6.1946 par Toumayeff G. (Linder 1953); ${ }^{2)} 1$ ex., Ofenpass, NP [National Park], 7.1971, par Scherler P. (Allenspach et Wittmer 1979).

Commentaire. E. ater est une espèce d'Europe centrale, incertaine en France (où elle n'a tout du moins plus été revue depuis longtemps, selon Constantin 2014). Pour la Suisse, les individus mentionnés dans la littérature ont pu être retrouvés mais leur identification était incorrecte : le spécimen de Versoix était un Hypebaeus flavipes, alors que celui de l'Ofenpass a été attribué à E. flavicornis (voir C54). Al'heure actuelle, nous n'avons donc aucune certitude quant à la présence de cette espèce en Suisse, bien qu'elle soit notamment citée du Sud-Tyrol italien (Kahlen et Hellrigl 1996).

\section{C52) Ebaeus (Ebaeus) battonii}

Matériel examiné. 1 ex., Chiasso, 26.5., coll. Fontana P., MHNG; 1 ex., Kt. Wallis, Martigny, leg. \& coll. Linder A., ETH; 1 ex., Grono, 7.1887, leg. Anonymous, NMBE; 1 ex., Castello, 2.7.1924, coll. Fontana P., MSNL; 1 ex., Chiasso, rsv [Riva San Vitale], 8.6.1925, coll. Fontana P., MSNL; 1 ex., Tessin, Mendrisio, 10.6.1944, leg. \& coll. Pochon H., MHNF; 1 ex., Mendrisio, 6.1950, leg. \& coll. Linder A., ETH; 1 ex., Kt. Tessin, Lugano, 4.1959, leg. \& coll. Linder A., ETH; 1 ex., Minusio TI, 9.-12.7.1965, leg. \& coll. Allenspach V., NMB; 1 ex., Mendrisio TI, 16.6.1971, leg. Allenspach V., MHNG; 1 ex., Tessin, Rovio, 7.7.1980, leg. \& coll. Scherler P., NMBE; 1 ex., Riva S. Vitale, 26.6.1984, leg. \& coll. Scherler P., NMBE; 1 ex., Riva S. Vitale, 4.7.1984, leg. \& coll. Scherler P., NMBE; 1 ex., Someo TI, 17.6.2008, leg. \& coll. Brägger H.

Commentaire. Cette espèce est connue du Bas-Rhin (Callot 2011) et du sud-est de la France (Constantin 2014), d'Italie, d'Autriche et de Suisse, où elle est signalée du Tessin principalement. Selon les critères donnés par Pardo Alcaide (1962) et Plata Negrache (2009), c'est bien cette espèce et non E. collaris (voir C53) qui est présente en Suisse.

\section{C53) [Ebaeus (Ebaeus) collaris collaris]}

Données publiées. ${ }^{1,8}$ St. Bernhard par Luisier (Stierlin 1883); ${ }^{2,8}$ Martigny par Favre (Favre 1890); ${ }^{2,8} 1$ ex., 
Chiasso, 1914 par Fontana P. [MSNL] (Fontana 1925); ${ }^{2,8}$ Riva St. Vitale par Fontana P. [MSNL] (Fontana 1947).

Commentaire. Cette espèce d'Europe méridionale est connue d'Autriche, de Croatie, du sud-ouest de la France, de Grèce, de Sardaigne, du Portugal, d'Espagne et de Russie. En suivant les clés d'identifications fournies par Pardo Alcaide (1962) et par Plata Negrache (2009), les spécimens de la littérature qui ont été retrouvés en collection ont été attribués à l'espèce voisine $E$. battonii, décrite en 1962 et donc postérieurement aux dates de publications citées ci-dessus. E. collaris n'est pas présent en Suisse.

\section{C54) Ebaeus (Ebaeus) flavicornis}

Matériel examiné. 1 ex., Fully, leg. Anonymous, det. Švihla V., MHNG; 2 ex., Tarasp, leg. Anonymous, BNM; 1 ex., Schuls GR, 7.1938, leg. Toumayeff G., det. Švihla V., MHNG; 1 ex., Ofenpass GR, 27.7.1971, leg. \& coll. Scherler P., NMBE; 1 ex., Sulgen, Langstuden TG, 24.7.1990, leg. Blöchlinger H., det. Liberti G., MHNG.

Données publiées. ${ }^{1)} 3$ ex., Umgebung Sihlsee, 6.1946 par Lautner J. (Linder 1968); 1)1 ex., IrchelgebietFreienstein ZH par Lautner J. (Allenspach et Wittmer 1979).

Commentaire. Seul un des spécimens examinés, de Tarasp dans les Grisons, est un mâle. Tous les autres spécimens sont des femelles et une confusion avec E. appendiculatus et $E$. ater (voir C50 et C51) n'est dès lors pas exclue. Au vu de la distribution générale d'E. flavicornis et de sa présence confirmée dans le sud de l'Allemagne (Köhler et Klausnitzer 1998) et en Alsace (Callot 2011), la donnée de Sulgen TG devrait néanmoins aussi se rattacher à cette espèce. Les spécimens cités dans la littérature n'ont pas été retrouvés dans la collection de Lautner J. au NMB.

\section{C55) Ebaeus (Ebaeus) gibbus}

Matériel examiné. 3 ex., Chiasso, 25.6., coll. Fontana P., MSNL; 1 ex., Chiasso, 26.6.1937, coll. Fontana P., MSNL; 1 ex., Chiasso, 21.7.1941, coll. Fontana P., MHNG; 1 ex., Kt. Tessin, Mendrisio, 6.1950, leg. \& coll. Linder A., ETH; 3 ex., Kt. Tessin, Rancate, 5.1968, leg. \& coll. Linder A., ETH; 2 ex., Kt. Tessin, Tremona, 7.1970, leg. \& coll. Linder A., ETH; 1 ex., Kt. Tessin, Arogno, 5.1971, leg. \& coll. Linder A., ETH.

Données publiées. ${ }^{1)}$ St. Bernhard par Stierlin G. (Stierlin 1883); Chiasso, 25.6. par Fontana P. (Fontana 1925).

Commentaire. Quelques spécimens collectés au Tessin confirment la présence en Suisse de cette espèce connue également d'Italie, de Corse et de Malte (Constantin 2014). L'espèce n'a toutefois plus été observée en Suisse depuis près de cinquante ans.

\section{C56) [Ebaeus (Ebaeus) cf. rufipes]}

Matériel examiné. ${ }^{3,8)} 1$ ex., Alpes, Tête Noire, 12.7., det. Liberti G., coll. Maerky C., MHNG.
Commentaire. Le spécimen examiné se rapproche d'E. rufipes, une espèce connue de Russie, d'Ukraine, du Kazakhstan et du Turkménistan. Provenant d'une collection problématique, il ne doit toutefois pas être considéré. Cette espèce ne fait pas partie de la faune suisse.

\section{C57) [Hypebaeus (Hypebaeus) flavicollis]}

Matériel examiné. ${ }^{3,8)} 2$ ex., Fully, 6.5.1929, coll. Gaud A., MZL.

Données publiées. ${ }^{3,8}$ Fully par Favre E. (Favre 1890).

Commentaire. Cette espèce méridionale est connue des Baléares, du sud de la France, de Corse, d'Italie, de Malte ainsi que d'Algérie. Malgré la présence de deux spécimens en collection, soutenus par une citation dans la littérature, $H$. flavicollis n'est pas considéré comme indigène au vu de sa distribution générale en Europe et en Afrique du Nord. La collection d'Alphons Gaud contient en effet ponctuellement des spécimens douteux, comme cela a déjà été mis en évidence pour d'autres espèces en Suisse (Monnerat et al. 2015a).

\section{C58) Malachius (Malachius) scutellaris}

Matériel examiné. 1 ex., London [Allondon], 16.5.1920, leg. Simonet J., MHNG; 1 ex., Kt. Freiburg, Freiburg, 5.1946, leg. \& coll. Linder A., ETH; 1 ex., London [Allondon], 11.5.1947, leg. Simonet J., MHNG; 6 ex., Genève, London [Allondon], 5.1949, leg. Toumayeff G., MHNG; 1 ex., Kt. Freiburg, Montbovon, 5.1949, leg. \& coll. Linder A., ETH; 1 ex., Ml. Vert [Moulin de Vert], 16.4.1957, leg. Anonymous, MHNG; 1 ex., Laconnex, 28.4.1957, leg. Anonymous, MHNG; 3 ex., Chancy, 7.5.1960, leg. Anonymous, MHNG; 1 ex., Chancy, La Laire, 17.5.1961, leg. Régnier J.-C., MHNG; 1 ex., Branson, 7.7.1963, leg. Van de Gümster J., MHNG; 1 ex., Genève, Russin, Allondon, 16.5.1996, leg. Besuchet C., MHNG.

Données publiées. ${ }^{1)}$ Genf par Böschenstein A., ${ }^{1)} \mathrm{Si}$ ders et ${ }^{1)}$ Sitten par Isenschmid M. (Stierlin 1883); ${ }^{1)} \mathrm{Ba}-$ sel et ${ }^{1)}$ Büren par Rätzer A. (Rätzer 1888); ${ }^{1}$ UnterWallis (Stierlin 1898).

Commentaire. Cette espèce colonise largement l'Europe occidentale, centrale et orientale. En Suisse, elle n'est présente que dans l'ouest du pays et principalement à Genève.

\section{C59) Micrinus heteromorphus}

Matériel examiné. 1 ex., Ticino, Locarno, Pizzo Trosa, leg. Focarile A., coll. Wittmer W., NMB; 1 ex., Mte. Lema Tic., 15.6.1955, leg. \& coll. Allenspach V., NMB; 3 ex., Indemini TI, 6.1968, leg. Toumayeff G., MHNG; 6 ex., A. di Neggia TI, 6.1968, leg. Toumayeff G., MHNG; 3 ex., Indemini TI, 7.1969, leg. Toumayeff G., MHNG \& NMB; 1 ex., A. di Neggia TI, 7.1971, leg. Toumayeff G., MHNG; 3 ex., Tessin, Pne di Breno, 17.7.1980, leg. \& coll. Scherler P., NMBE; 2 ex., Tessin, Mte. Gambarogno, 23.7.1980, leg. \& coll. Scherler P., NMBE; 2 ex., Pne 
di Breno TI, 29.6.1983, leg. \& coll. Scherler P., NMBE; 1 ex., Mte. Gambarogno TI, 23.6.1986, leg. \& coll. Scherler P., NMBE; 1 ex., Gambarogno TI, 19.6.2013, leg. \& coll. Szallies A.; 1 ex., Gambarogno TI, 4.7.2013, leg. \& coll. Szallies A.; 2 ex., Val Müstair GR, 19.7.2016 et 24.6.2017, leg. \& coll. Chittaro Y.

Commentaire. Cette espèce alpine est connue d'Italie, de France et de Suisse, où elle colonise quelques sommets du Tessin et du Val Müstair dans l'est des Grisons.

\section{C60) Nepachys cardiacae}

Fig. 1E

Matériel examiné. 2 ex., Mont. [Montana], coll. Berhaut J., MHNS; 1 ex., Kt. Wallis, Montana, [leg. Cerutti N.], coll. Linder A., ETH; 2 ex., Wallis, Montana, leg. \& coll. Cerutti N., MHNF; 3 ex., St. Luc, 8.1925, leg. Linder A., coll. Linder A. \& Wittmer W., ETH \& NMB; 1 ex., Graubünden, Alter Schynweg, 7.7.1936, leg. \& coll. Wolf J. P., ETH; 1 ex., Kt. Wallis, Bürchen, 7.1963, leg. \& coll. Linder A., ETH; 1 ex., Randonne s/Saillon VS, 1.8.1982, leg. \& coll. Scherler P., NMBE; 1 ex., Anniviers VS, 7.7.13.8.2014, leg. \& coll. Sanchez A.; 1 ex., Vollèges VS, 4.-17.7.2017, leg. \& coll. Sanchez A.; 1 ex., Simplon VS, 11.7.2018, leg. \& coll. Chittaro Y.

Données publiées. ${ }^{1)}$ Pontresina par von Heyden L. (Heyden 1864); ${ }^{1}$ Churwalden par Huguenin G. (Stierlin 1883); ${ }^{1)}$ Basel (Stierlin 1898); Montana par Cerutti N. (Linder 1941); ${ }^{1}$ Blockhaus Cluozza, 20.7.1921, ${ }^{1)}$ Plaun Larschaida, 24.8.1936 et ${ }^{1)}$ Zernez, Clüs, 26.7.1921 par Handschin E. (Handschin 1963); ${ }^{1)} 1$ ex., Zürichberg, 5., MZL (Allenspach et Wittmer 1979); 2 ex., Valais, Mayens de Sion, leg. Carret A., coll. Constantin R. (Constantin 2013).

Commentaire. Décrite de Scandinavie et assez répandue dans le nord de l'Europe, cette espèce est présente aussi sporadiquement dans certaines régions montagneuses de France et d'Europe centrale, dont les Alpes, où elle est rare (Constantin 2013). En Suisse, elle n'est connue que de quelques localités du Valais et des Grisons.

\section{C61) Sphinginus coarctatus}

Matériel examiné. 3 ex., Chiasso, 20.6., coll. Fontana P., MSNL; 1 ex., Kt. Tessin, Mendrisio, 6.1943, leg. \& coll. Linder A., ETH; 1 ex., Tessin, Mte. San Giorgio, 5.8.1970, leg. \& coll. Scherler P., NMBE; 1 ex., Kt. Tessin, Locarno, 5.1971, leg. \& coll. Linder A., ETH; 3 ex., Tessin, Mte. San Giorgio, 14.7.1980, leg. \& coll. Scherler P., NMBE; 1 ex., Mte. Ceneri TI, 28.6.1986, leg. \& coll. Scherler P., NMBE; 1 ex., Origlio TI, 24.6.1987, leg. \& coll. Scherler P., NMBE; 1 ex., Tessin, Lago di Muzzano, 24.6.1987, leg. Besuchet C., MHNG; 1 ex., Pne. d'Arzo TI, 25.6.1990, leg. \& coll. Scherler P., NMBE; 1 ex., Tessin, S. Salvatore, 17.6.1995, leg. Besuchet C., MHNG; 1 ex., Meride, Serpiano, 11.-20.7.1995, leg. Reser-Rezbanyai L., NMLU; 1 ex., Meride, Serpiano, 1.-10.7.1996, leg. Reser-Rezbanyai L., NMLU; 3 ex., Terre di Pedemonte TI, 13.5.2014, leg. \& coll. Chittaro Y.
Données publiées. ${ }^{1)}$ Monte Generoso par FreyGessner E. (Stierlin 1883).

Commentaire. Plusieurs spécimens tessinois attestent de la présence en Suisse de cette espèce d'Europe centrale et méridionale et du Maghreb. L'espèce voisine $S$. lobatus colonise quant à elle l'ouest et le nord de la Suisse.

\section{C62) [Troglops cephalotes]}

Données publiées. ${ }^{2)}$ Chiasso, 20.5., 4.6. par Fontana P. (Fontana 1925); ${ }^{2}$ Mendrisio, 6.1936 par Allenspach V. et ${ }^{2)} 1$ ex., Locarno, 7.1944 par Linder A. (Linder 1946); ${ }^{2)}$ Frasco (Fontana 1947); ${ }^{1)} 1$ ex., Finsterhennen BE, 10.1936, NMBE (Allenspach et Wittmer 1979); ${ }^{1)}$ Cevio TI, 2003 par Focarile A. (Focarile 2003).

Commentaire. Après vérification des identifications, tous les spécimens cités dans la littérature qui ont été retrouvés étaient en réalité des $T$. silo. Le très rare $T$. cephalotes, connu d'Autriche, de France et d'Italie, reste néanmoins possible en Suisse mais sa présence demande confirmation.

\section{C63) Phloiophilus edwardsii}

Matériel examiné. 3 ex., Genève, Vandoeuvres, 5.6.1992, leg. Besuchet C., MHNG.

Commentaire. Assez largement répandue, cette espèce est néanmoins assez rare en Europe centrale et occidentale (Kolibáč 2018). C'est aussi le cas en Suisse, où elle n'est connue que de trois spécimens capturés dans une localité genevoise. L'espèce étant principalement active en automne et en hiver, la date de capture tardive des spécimens suisses est étonnante. $P$. edwardsii se développe dans les carpophores du champignon Peniophora quercina notamment (Sanchez et al. 2018).

\section{C64) [Aplocnemus (Aplocnemus) albipilis]}

Matériel examiné. ${ }^{3,8} 1$ ex., Alpes, Novel, 27.6., leg. \& coll. Maerky C., MHNG; ${ }^{3,8} 1$ ex., Alpes, St. Bernard, 2.8., leg. \& coll. Maerky C., det. Constantin R., MHNG; ${ }^{3,8)} 1$ ex., Genève, Peney, 7.7., leg. \& coll. Maerky C., det. Constantin R., MHNG.

Commentaire. Les spécimens suisses examinés proviennent tous d'une collection problématique et ne doivent pas être considérés. Cette espèce ne colonise que la Péninsule ibérique et les Pyrénées-orientales françaises.

\section{C65) Aplocnemus (Aplocnemus) chalconatus}

Matériel examiné. 1 ex., Chiasso, coll. Fontana P., MSNL; ${ }^{8} 1$ ex., Trient, leg. Anonymous, coll. Kiener S., det. Majer K., MHNG; ${ }^{3)} 1$ ex., Trient, leg. Preudhomme de Borre C., MHNG; 1 ex., Mendrisio, 5.1943, leg. \& coll. Linder A., ETH; 2 ex., Mendrisio, 6.1943, leg. \& coll. Linder A., ETH; 1 ex., Tessin, Mendrisio, 1.6.1944, leg. \& coll. Pochon H., det. Constantin R., MHNF; 2 ex., V. Calanca, 5.6.1948, leg. \& coll. Allenspach V., NMB; 
2 ex., Mendrisio, 5.1949, leg. \& coll. Linder A., ETH; 1 ex., Mendrisio, 16.-25.6.1949, leg. \& coll. Allenspach V., NMB; 2 ex., Mendrisio, 6.1949, leg. \& coll. Linder A., ETH; 1 ex., Mendrisio, 6.1950, leg. \& coll. Linder A., ETH; 4 ex., Brusio GR, 20.-21.6.1967, leg. \& coll. Allenspach V., NMB; 1 ex., Campascio GR, 30.7.1969, leg. \& coll. Scherler P., det. Constantin R., NMBE; 1 ex., Miralago GR, 18.7.1970, leg. \& coll. Scherler P., det. Constantin R., NMBE; 1 ex., Cavaione GR, 9.7.1975, leg. \& coll. Scherler P., NMBE; 1 ex., Brusio GR, 7.1975, leg. Toumayeff G., MHNG; 1 ex., Poschiavo GR, Zalende, 18.7.1984, leg. Besuchet C., MHNG; 1 ex., Lago di Poschiavo, 1.6.1990, leg. \& coll. Brägger H.; 2 ex., Brusio GR, 17.5.-16.6.2012, leg. Sanchez A., det. Geiser M., coll. Chittaro Y.; 1 ex., Brusio GR, 16.6.-10.07.2012, leg. Sanchez A., det. Geiser M., coll. Geiser M., NMBE.

Données publiées. ${ }^{1,8)}$ Schaffhausen par Stierlin G. (Stierlin et Gautard 1867); ${ }^{1,8}$ St. Gallen par Müller J. A. (Müller 1904); Chiasso et ${ }^{1)}$ Generoso par Fontana P. (Fontana 1925); ${ }^{1}$ Bisbino par Fontana P. (Fontana 1947); ${ }^{2}$ Wallis, ETH (Allenspach et Wittmer 1979).

Commentaire. Largement répandue en Italie, en Autriche et dans la Péninsule balkanique, et marginale en France, cette espèce floricole atteint sa limite septentrionale de répartition dans le sud de la Suisse (Tessin et Grisons). Les citations de la littérature de Schaffhausen et de St. Gallen sont invérifiables mais hautement douteuses. Quant à celle du Valais (Wallis), il s'agissait en réalité d'A. tarsalis.

\section{C66) Aplocnemus (Aplocnemus) integer}

Matériel examiné. 5 ex., Chiasso, coll. Fontana, MSNL; 3) 1 ex., Bienne, 29.6.1908, coll. Maerky C., det. Majer K., MHNG; 1 ex., Chiasso, 1932, coll. Burghold W., det. Liberti G., NMBE; 1 ex., Mendrisio, 6.1943, leg. \& coll. Linder A., ETH; 1 ex., Mendrisio, 6.1949, leg. \& coll. Linder A., ETH; 1 ex., Somazzo, Toretta-O. TI, 16.5.1990, leg. Reser-Rezbanyai L., det. Liberti G., NMLU.

Commentaire. Cette espèce d'Europe centrale et méridionale n'est connue en Suisse que de quelques spécimens valides, tous provenant du sud du Tessin. L'individu de Bienne est issu d'une collection problématique et ne doit pas être considéré. C'est le plus rare de nos Aplocnemus.

\section{C67) [Aplocnemus (Aplocnemus) ramicornis]}

Matériel examiné. ${ }^{3,8)} 1$ ex., Genève, Aïre, 10.5., leg. \& coll. Maerky C., det. Constantin R., MHNG; ${ }^{3,7,8)} 1$ ex., Jura, Dôle, 2.7., coll. Maerky C., det. Majer K., MHNG.

Commentaire. Cette espèce n'est connue que d'Ukraine, ce qui discrédite une fois encore la collection à laquelle appartiennent les spécimens « suisses » examinés.

\section{C68) [Trichoceble floralis]}

Données publiées. ${ }^{1)}$ Puschlav par Killias E. (Killias 1860); ${ }^{1)}$ Genf par Chevrier F., ${ }^{1)}$ Engadin et ${ }^{1)}$ Monte Rosa par Gautard V. (Stierlin et Gautard 1867); ${ }^{1)}$ Entremont par Rätzer A. (Rätzer 1888); ${ }^{1)}$ Sussillon par Favre E. (Favre 1890); ${ }^{1)}$ Pontresina par Meyer-Dür R. (Stierlin 1863-1864); 1)1 ex., Pfyn-Sierre, 5.1919, NMB, ${ }^{2} 1$ ex., V. d'Hérens, Euseigne par Allenspach V. et ${ }^{2)} 1$ ex., V. d'Anniviers, Ayer, 7.1961 par Scherler P. (Allenspach et Wittmer 1979).

Commentaire. T. floralis est une espèce qui présente une distribution européenne assez large mais discontinue. Elle est sporadique dans la majeure partie de la France et semble inféodée aux vieilles futaies (Constantin et Liberti 2011). Aucun spécimen de référence n'existe pour la Suisse, la plupart des spécimens cités dans la littérature n'ayant pas été retrouvés à l'exception de deux (ceux d'Euseigne et d'Ayer) qui étaient des T. memnonia (C69). Citée d'Alsace (Callot 2018) et du sud de l'Allemagne (Köhler et Klausnitzer 1998), T. floralis pourrait néanmoins aussi se trouver en Suisse.

\section{C69) Trichoceble memnonia}

Matériel examiné. 1 ex., Simplon VS, 7.1881, coll. Rätzer A., NMBE; ${ }^{8} 1$ ex., Kt. Bern, Biel, 6.1913, leg. \& coll. Linder A., ETH; 1 ex., Val. [Valais], Euseigne, 7.18.7.1941, leg. \& coll. Allenspach V., NMB; 1 ex., Valais, Ayer, 3.8.1961, leg. \& coll. Scherler P., NMBE; 1 ex., Kt. Wallis, Bürchen, 8.1963, leg. \& coll. Linder A., ETH; 2 ex., Kt. Graubünden, Felsberg, 7.1971, leg. \& coll. Linder A., ETH; 1 ex., Ilanz GR, 1.7.1971, leg. \& coll. Pochon H., MHNF; 1 ex., Bürchen VS, 13.7.1974, leg. \& coll. Scherler P., NMBE.

Données publiées. 1 ex., Annivier-Thale, 1861 par Kiesenwetter H. (Kiesenwetter 1861); 1 ex., Bürchen, 8.1963 par Linder A. (Linder 1968); 1 ex., Briey-Chalais-Chippis VS, 7. 1975 par Toumayeff G., ${ }^{1)} 2$ ex., Palagnedra-Verdasio-Centovalli TI, 7.1976 par Besuchet C. (Allenspach et Wittmer 1979), 1 ex., Sion, 1903, par Robert (Liberti 2012).

Commentaire. Décrite sur la base d'une femelle du Val d'Anniviers (Kiesenwetter 1861), cette espèce rare d'Europe occidentale et centrale est inféodée aux étages montagnards et subalpins (Constantin et Liberti 2011). En Suisse, elle est connue de quelques données isolées provenant de localités alpines, mais n'a plus été signalée depuis près de cinquante ans. Nous n'avons pas retrouvé l'exemplaire tessinois capturé en 1976 cité dans Allenspach et Wittmer (1979), et considérons la localité de Biel [Bienne], sur le Plateau suisse, comme problématique.

\section{C70) [Thaneroclerus buquet]}

Matériel examiné. 1 ex., Kirchberg SG, 17.3.2009, leg. Anonymous, det. Landau-Lüscher I., SPZH; Dinhard ZH, 3.4.2009, leg. Anonymous, det. Schmidt M., SPZH; Zürich ZH, 11.2.2010, 20.2.2012 et 24.2.2016, leg. Anonymous, det. Schmidt M., SPZH; Uster ZH, 21.10.2011, leg. Anonymous, SPZH; Wangen-Brüttisellen ZH, 6.3.2012, leg. Anonymous, det. Schmidt M., SPZH; 2 ex., Zug ZG, 27.3.2013, leg. Anonymous, det. 
Landau-Lüscher I., SPZH; Les Genevez JU, 2.10.2013, leg. Anonymous, det. Schmidt M., SPZH; Dietlikon ZH, 30.10.2013, leg. Anonymous, det. Schmidt M., SPZH.

Commentaire. Probablement originaire d'Asie du sud-est ou d'Inde, cette espèce synanthrope est maintenant presque cosmopolite. Quelques larves ont été trouvées en Suisse au cours des dernières années dans plusieurs localités et soumises pour identification au centre de Schädlingsprävention und -beratung de Zürich. Leur importation accidentelle en Suisse ne fait aucun doute, comme c'est aussi le cas en Allemagne (Niehuis 2013). T. buquet est un prédateur de Coléoptères, Ptinidae et Tenebrionidae notamment, présents dans les denrées stockées (thé, café, riz, tabac, ...).

\section{C71) Grynocharis oblonga}

Matériel examiné. ${ }^{3)} 1$ ex., Alpes, Tessin, coll. Maerky C., ex coll. Ghidini A., MHNG; ${ }^{3)} 1$ ex., Alpes, Aigle, 7. , coll. Maerky C., MHNG; ${ }^{3} 2$ ex., Genf, coll. Lasserre H., ETH; 1 ex., Osco, Leventina (Predelp), leg. \& coll. Focarile A.; ${ }^{3)} 1$ ex., Valais, 7.6.1886, coll. Gaud A., MZL; 6) 1 ex., Kt. Luzern, Hünenbergstr., 3.1946, leg. \& coll. Pochon H., MHNF; 1 ex., Bollingen SG, 9.5.1967, leg. \& coll. Spälti A., MHNG.

Données publiées. ${ }^{6} 1$ ex., Luzern (vermutlich eingeschleppt) par Pochon H. (Linder 1953).

Commentaire. Très largement répandue à l'échelle européenne, cette espèce n'est pourtant connue que de rares données en Suisse, parmi lesquelles seulement deux (d'Osco au Tessin et de Bollingen à Saint-Gall) paraissent fiables. Quant à la mention de Lucerne, elle est vraisemblablement issue d'une importation (Linder 1953).

\section{C72) Calitys scabra}

Matériel examiné. ${ }^{3)} 1$ ex., Alpes, Tessin, coll. Maerky C., ex coll. Ghidini A., MHNG; ${ }^{3)} 1$ ex., Alpes, Valais, coll. Maerky C., MHNG; 2 ex., Doml. [Domleschg], coll. Jörger J. J., ex coll. Killias E., NMB; 1 ex., Domleschg GR, coll. Killias E., MHNG; 1 ex., Furstenau, leg. Anonymous, NMAA; 1 ex., Helvetia, coll. Burghold W., NMBE; ${ }^{3)} 1$ ex., Kt. Wallis, coll. Linder A., ex coll. Täschler M., ETH; ${ }^{3)} 1$ ex., Ober-Wallis, coll. Heer O., ETH; 1 ex., Valais, coll. Mellet L., MZL; ${ }^{3,8} 1$ ex., Weissb. [Weissenburg], coll. Huguenin G., ETH; ${ }^{3} 1$ ex., Alpes, Viège, 4.7., coll. Maerky C., MHNG; ${ }^{3)} 1$ ex., Genève, Aïre, 10.5., coll. Maerky C., MHNG; 1 ex., Rätia, Rezia? [Canton des Grisons], 1885, leg. Anonymous, MHNG; 1 ex., Saas, 7.1886, leg. Anonymous, NMBE; 1 ex., V. Somvix [Val Sumvitg], 25.7.1891, leg. Anonymous, NMBE.

Données publiées. ${ }^{1)}$ Vallée de Conches bei Visp im Wallis par Venetz I. et ${ }^{1)}$ Oberwallis par Gautard V. (Stierlin et Gautard 1867); ${ }^{1)}$ Chur par Scheuchzer (Stierlin 1883); Saas par Steck-Hofmann T. et ${ }^{1)}$ Simplon par Favre E. (Favre 1890); ${ }^{1}$ Domleschg par Mengold, ${ }^{1)}$ Malans par Amstein J.R. et ${ }^{1)}$ Thusis par Amstein J. R. (Caflisch 1894); ${ }^{1)}$ Bérisal par Bourgeois J. (Bourgeois 1909).
Commentaire. Cette espèce colonise la zone boréale et les montagnes de toute l'Europe mais est généralement très rare. En Suisse, elle est connue uniquement de quelques données très anciennes et n'y a plus été retrouvée depuis plus d'un siècle. Elle est liée aux polypores du genre Antrodia, notamment Antrodia xantha (Brustel 2009).

\section{C73) Nemozoma caucasicum}

Matériel examiné. Nombreux exemplaires du Plateau suisse principalement, mais aussi du Tessin et du Chablais vaudois.

Données publiées. 1 ex., Dardagny GE, 22.7.10.8.2012 par Blanc M. (Chittaro et al. 2013).

Commentaire. Originaire du Caucase, cette espèce est en forte expansion en Europe occidentale (Kolibáč 2018). Elle a été signalée pour la première fois en Suisse à Genève en 2012 (Chittaro et al. 2013), puis dans le nord du pays (en Argovie) en 2013 et dans le sud du Tessin en 2018. Elle s'étend maintenant dans notre pays à partir de ces trois voies de colonisation.

\section{C74) [Nemozoma cornutum]}

Données publiées. ${ }^{1,8)}[$ Suisse] par Bremi-Wolf J. J. (Bremi-Wolf 1856).

Commentaire. La citation de la littérature, sans aucune précision, se réfère assurément à une autre espèce. $N$. cornutum n'est connu que du sud de la Russie et d'Ukraine et ne fait donc pas partie de notre faune.

\section{C75) Tenebroides mauritanicus}

Matériel examiné. Nombreux exemplaires, de la plupart des régions de Suisse.

Commentaire. A l'instar de ce qui a été proposé par Kolibáč (2007, 2013), et bien qu'il existe certaines différences morphologiques entre les deux taxa (Kolibáč 1993), nous avons considéré ici sous T. mauritanicus non seulement les spécimens synanthropes, mais également les spécimens trouvés in natura qui sont parfois attribués à une espèce distincte: T. fuscus Goeze, 1777. La présence des deux taxa est dans tous les cas attestée en Suisse (Huber et Kobel 1994, Herger 1998a).

\section{Discussion}

Cette liste commentée s'inscrit dans la continuité des synthèses réalisées au cours des dernières années sur diverses familles de Coléoptères (Marggi et Luka 2001, Carron 2005, Carron 2008, Luka et al. 2009, Germann 2010, Chittaro et Blanc 2012, Reibnitz et al. 2013, Monnerat et al. 2015b, Breitenmoser et al. 2016, Chittaro et Sanchez 2016b, Cosandey et al. 2017, Sanchez et Chittaro 2018) et contribue à améliorer nos connaissances sur la faune de Suisse. Elle fournit, avec les cartes de distribution basées sur les données récoltées (disponibles sur le serveur 
Table 1. Nombre d'espèces de Cleridae, Melyridae et Rhadalidae, considérées comme résidentes en Suisse par Allenspach and Wittmer (1979) et par le présent travail, par famille. Les espèces supprimées (-) et ajoutées (+) par rapport à la liste de 1979 sont indiquées.

\begin{tabular}{|c|c|c|c|c|}
\hline & $\begin{array}{l}\text { Allenspach and Wittmer } \\
1979\end{array}$ & - & + & $\begin{array}{c}\text { Présent travail } \\
2019 \\
\end{array}$ \\
\hline CLERIDAE & 20 & $\begin{array}{c}\text { Opilo germanus } \\
\text { Tarsostenus univittatus } \\
\text { Trichodes favarius } \\
\text { Trichodes ircutensis }\end{array}$ & $\begin{array}{l}\text { Denops albofasciatus } \\
\text { Korynetes ruficornis }\end{array}$ & 18 \\
\hline MELYRIDAE & 71 & $\begin{array}{c}\text { Anthomalachius spinosus } \\
\text { Attalus amictus } \\
\text { Attalus cyaneus } \\
\text { Ceratistes dilaticornis } \\
\text { Clanoptilus barnevillei } \\
\text { Clanoptilus rufus } \\
\text { Clanoptilus spinipennis } \\
\text { Danacea marginata } \\
\text { Ebaeus appendiculatus } \\
\text { Ebaeus ater } \\
\text { Hypebaeus flavicollis }\end{array}$ & $\begin{array}{c}\text { Anthocomus humeralis } \\
\text { Charopus docilis } \\
\text { Danacea montivaga } \\
\text { Dasytes pauperculus } \\
\text { Dasytes thoracicus } \\
\text { Malachius australis }\end{array}$ & 66 \\
\hline RHADALIDAE & 9 & Trichoceble floralis & & 8 \\
\hline Total & 100 & 16 & 8 & 92 \\
\hline
\end{tabular}

cartographique d'info fauna - CSCF, http://www.cscf.ch), une synthèse des connaissances actuelles sur les espèces appartenant à la superfamille des Cleroidea. Sept familles de Cleroidea sont actuellement attestées de Suisse, mais une huitième pourrait être encore découverte dans le futur, à savoir celle des Acanthocnemidae par le biais de l'espèce pyrophile cosmopolite Acanthocnemus nigricans (Hope, 1845), originaire d'Australie, mais introduite et déjà établie en France et en Italie (Constantin 2014, Kolibáč 2018).

En comparant notre liste avec la synthèse d'Allenspach et Wittmer (1979) pour les trois familles communes aux deux travaux, il apparaît que les chiffres annoncés en terme d'espèces par famille sont légèrement différents: alors qu'Allenspach et Wittmer (1979) annonçaient 100 espèces de Cleridae, Melyridae et Rhadalidae, nous en considérons pour notre part 92 comme suisses (Tab. 1). Si huit espèces ont été ajoutées depuis 1979, 16 autres, qu'Allenspach et Wittmer (1979) considéraient comme suisses, ont ainsi été écartées. La raison en est principalement le nouvel éclairage apporté par Monnerat et al. (2015a) sur plusieurs collections que l'on sait maintenant problématiques (voir commentaires sur ces espèces).

Si nos connaissances sur la répartition des espèces appartenant à la superfamille des Cleroidea peuvent être qualifiées de bonnes en Suisse, ce n'est pas le cas de nos connaissances sur leur écologie, qui demeurent encore très lacunaires et notamment chez les Melyridae. Si quelques espèces se développent dans des plantes herbacées à grande tige (Psilothrix spp., Dolichosoma lineare) ou se rencontrent sous des pierres (Enicopus spp. et Danacea spp.) (Constantin et Liberti 2011), il est néanmoins admis que la majorité des Cleroidea sont saproxyliques. Leurs larves se développent sous des écorces ou dans un substrat ligneux plus ou moins décomposé, souvent en tant que prédateurs (ou prédateurs occasionnels) d'autres organismes. Quelques uns sont mycétophages (Peltinae notamment). Plusieurs Cleroidea tels Allonyx quadrimaculatus (Fig. 1A) et Peltis grossa (Fig. 1F) présentent des exigences écologiques très élevées, et figurent sur la liste des Coléoptères saproxyliques emblématiques de Suisse (Sanchez et al. 2016). Certains représentants des Melyridae et des Rhadalidae (Dasytes nigrocyaneus, $D$. pauperculus, Aplocnemus integer, Trichoceble memnonia notamment,...), deux familles qui n'ont pas encore été traitées par Sanchez et al. (2016), devraient y figurer également lors d'une future actualisation.

Du point de vue conservatoire, signalons que bon nombre de ces espèces forestières se sont fortement raréfiées en Suisse au cours du siècle dernier et certaines (Calitys scabra, Trichoceble memnonia notamment) n'ont plus été trouvées dans notre pays depuis très longtemps. Les espèces liées aux milieux ouverts séchards ont également vu leurs populations décroître en même temps que disparaissaient leurs habitats. Très largement répandu par le passé, Clanoptilus marginellus n'a ainsi plus été signalé en Suisse au cours des vingt dernières années. Les espèces du genre Charopus semblent également s'être fortement raréfiées. Ce bilan peu réjouissant ne va $a$ priori pas aller en s'améliorant, au vu de la poursuite d'une urbanisation galopante (Lachat et al. 2010) sur les rares milieux encore favorables. Les espèces qui colonisent les étages subalpins et alpins sont un peu mieux loties et profitent de milieux généralement mieux conservés. C'est par exemple le cas de Dasytes lombardus, D. thoracicus, Danacea denticollis, D. montivaga, Micrinus heteromorphus et Attalus alpinus, autant d'espèces endémiques des Alpes. La Suisse porte une responsabilité élevée pour leur conservation! 


\section{Remerciements}

Cette liste commentée a largement profité des travaux initiés par Claude Besuchet $(\mathrm{CH}$ - Genève) dans le cadre de son projet de Catalogue des Coléoptères de Suisse, et notamment de l'important travail de révision des collections suisses effectué par plusieurs spécialistes européens: Robert Constantin (F-Saint-Lô), feu Karel Majer (CZ-Brno) et feu Vladimír Švihla (CZ-Prague). Qu'ils en soient ici chaleureusement remerciés!

Un immense merci à Gianfranco Liberti (I-Uboldo) pour son aide tout au long du projet, notamment la vérification de plusieurs spécimens problématiques et pour sa relecture critique du manuscrit. Merci aussi à Stève Breitenmoser (CH-Givrins), à Robert Constantin (F-Saint-Lô), à Benoît Dodelin (F-Lyon), à Gabriele Franzini (I-Milano), à Roland Gerstmeier (D-Freising), à Jiř́ Kolibáč (CZ-Brno), à Ivan Löbl (F-Genève), à Christian Monnerat (CH-Neuchâtel) et à Ulrich Schneppat (CH-Malix) pour leurs commentaires précieux, ainsi qu'à Georg Friebe (A-Dornbirn) et à Ulrich Hiermann (A-Rankweil) pour leurs apports bibliographiques.

Nous tenons également à remercier ici l'ensemble des conservateurs des musées de Suisse qui nous ont si souvent et si aimablement accueillis au sein de leurs institutions aux cours de ces dernières années, ainsi que tous les coléoptéristes actifs sur ce groupe qui, par la transmission de leurs observations personnelles, ont permis d'enrichir les connaissances sur la distribution de ces espèces en Suisse. Un grand merci également à Gaspard Braulin (CH-Lausanne) et à Arnaud Vallat (CH-Neuchâtel) pour leur aide précieuse apportée lors de la saisie des spécimens des collections muséales consultées, ainsi qu'à Giulio Cuccodoro (MHNG) et à Michel Sartori (MZL) pour nous avoir aimablement mis à disposition leur matériel photographique.

\section{Références}

Allenspach V (1968) Käferfang am Schmetterlingsschirm in Minusio/TI Mitteilungen der Entomologischen Gesellschaft Basel 18: 125-133.

Allenspach V (1971) Käferfang am Schmetterlingschirm in Minusio/ Tessin (Erster Nachtrag). Mitteilungen der Entomologischen Gesellschaft Basel 21: 110-114.*

Allenspach V, Wittmer W (1979) Cantharoidea, Cleroidea, Lymexylonoidea. Insecta Helvetica 4, 139 pp.

Besuchet C (1983) Coléoptères des Alpes suisses atteignant ou dépassant l'altitude de 3000 m. Bulletin Romand d'Entomologie 1(3): 167-176.*

Bocák L, Barton C, Crampton-Platt A, Chester D, Ahrens D, Vogler AP (2014) Building the Coleoptera tree-of-life for $>8000$ species: composition of public DNA data and fit with Linnaean classification. Systematic Entomology 39: 97-110. https://doi.org/10.1111/ syen. 12037

Bocáková M, Constantin R, Bocák L (2012) Molecular phylogenetics of the melyrid lineage (Coleoptera: Cleroidea). Cladistics 28: 117-129. https://doi.org/10.1111/j.1096-0031.2011.00368.x

Bouchard P, Bousquet Y, Davies A, Alonso-Zarazaga M, Lawrence J, Lyal C, Newton A, Reid C, Schmitt M, Slipinski A, Smith A (2011)
Family-group names in Coleoptera (Insecta). ZooKeys 88: 1-972. https://doi.org/10.3897/zookeys.88.807

Bourgeois J (1909) Notes sur quelques espèces de Coléoptères de la faune alpine. Mitteilungen der Entomologischen Gesellschaft 11: 388-395.

Brandstetter CM, Kapp A (1998) Käferinventar von Vorarlberg und Liechtenstein. Insecta: Coleoptera. Eigenverlag des Ersten Vorarlberger Coleopterologischen Vereins, Bürs, 92 pp.

Breitenmoser S (2017) Les chênes séculaires de Duillier (VD): un sanctuaire pour des Coléoptères saproxyliques exceptionnels. Bulletin de la Société Vaudoise des Sciences Naturelles 96: 49-76.*

Breitenmoser S, Chittaro Y, Sanchez A (2016) Liste commentée des Oedemeridae (Coleoptera) de Suisse. Mitteilungen der Schweizerischen Entomologischen Gesellschaft 89: 73-92.

Bremi-Wolf JJ (1856) Catalog der Schweizerischen Coleopteren, als Vorläufer der Beiträge für Schweizerische Entomologie. Druck und Commission von Friedrich Schulthess, Zürich, 78 pp.

Brustel H (2009) Antrodia spp., Polypores hôtes de rares Quilnus spp. (Heteroptera Aradidae) et de Calytis scabra (Coleoptera Trogositidae) et révélation d'un haut lieu entomologique dans les Pyrénées: la vallée du Rioumajou. L'Entomologiste 65(5): 226-232.

Bugnion E (1880) Notes sur les coléoptères des Alpes vaudoises. Jahrbuch des Schweizer Alpenclub 16: 109-120.*

Caflisch JL (1894) Beiträge zu einem Verzeichnisse der Insecten-Fauna Graubündens von Dr. E. Killias. IV: Coleopteren. Supplement/Beilage zu Jahresbericht der Naturforschenden Gesellschaft Graubündens, 275 pp.

Callot H (2011) Cantharis paradoxa Hicker, 1960, Ebaeus flavicornis Erichson, 1840 et E. battonii Pardo, 1962, trois espèces probablement nouvelles pour la faune de France. Rappel de la présence d'une autre espèce méconnue, Olibrus gerhardti Flach, 1888, dans les Vosges (Coleoptera Cantharidae, Malachiidae et Phalacridae). L'Entomologiste 67(3): 121-123.

Callot H (2018) Liste de référence des Coléoptères d'Alsace. Société Alsacienne d'Entomologie, 107pp. http://soc.als.entomo.free.fr [version du 30-IX-2018]

Carron G (2005) Kommentierte Checkliste der Dytiscidae und Noteridae (Coleoptera) der Schweiz. Mitteilungen der Entomologischen Gesellschaft Basel 55 (3): 93-114.

Carron G (2008) Checklist des coléoptères aquatiques de Suisse. Deuxième partie: Gyrinidae, Haliplidae, Paelobiidae, Sphaeriusidae. Mitteilungen der Schweizerischen Entomologischen Gesellschaft 81: 53-60.

Chittaro Y (2014) Denops albofasciatus (Charpentier, 1925), un Cleridae (Coleoptera) nouveau pour la Suisse. Entomo Helvetica 7: 145-146.

Chittaro Y, Blanc M (2012) Liste commentée des Cerophytidae, Elateridae, Eucnemidae et Throscidae (Coleoptera) de Suisse. Mitteilungen der Schweizerischen Entomologischen Gesellschaft 85: 91-114.

Chittaro Y, Sanchez A (2016a) Inventaire des Coléoptères saproxyliques d'un site exceptionnel: la Châtaigneraie de Fully (VS). Bulletin de la Murithienne 133: 13-27.*

Chittaro Y, Sanchez A (2016b) Liste commentée des Tenebrionoidea (Coleoptera) de Suisse. Partie 1: Aderidae, Anthicidae, (Boridae), Melandryidae, Meloidae, Mycetophagidae, Mycteridae, Prostomidae, Pyrochroidae, Pythidae, Ripiphoridae, Salpingidae, Tenebrionidae, Tetratomidae, Zopheridae. Mitteilungen der Schweizerischen Entomologischen Gesellschaft 89: 183-235.

Chittaro Y, Sanchez A (2017) A propos de quelques Coléoptères rares ou nouveaux pour la Suisse. Entomo Helvetica 10: 45-53. 
Chittaro Y, Sanchez A, Blanc M, Monnerat C (2013) Coléoptères capturés en Suisse par pièges attractifs aériens: bilan après trois années et discussion de la méthode. Entomo Helvetica 6: 101-113.

Constantin R (2013) Contribution à la connaissance des Malachiidae d'Europe et description de deux espèces nouvelles (Coleoptera, Cleroidea). Bulletin de la Société entomologique de France 118(3): 305-320.

Constantin R (2014) Dasytidae, Acanthocnemidae, Prionoceridae, Melyridae, Malachiidae. pp. 468-477. In: Tronquet M (Coord) Catalogue des Coléoptères de France. Perpignan, Association Roussillonnaise d'Entomologie, $1056 \mathrm{pp}$.

Constantin R, Liberti G (2011) Coléoptères Dasytidae de France. Musée des Confluences, Lyon, 144 pp.

Cosandey V, Chittaro Y, Sanchez A (2017) Liste commentée des Scarabaeoidea (Coleoptera) de Suisse. Alpine Entomology 1: 5790. https://doi.org/10.3897/alpento.1.21179

Crowson RA (1955) The natural classification of the families of Coleoptera. Nathaniel Llyod, Co., Ltd., London, 187 pp.

Dietrich K (1865) Beitrag zur Kenntnis der Insekten-Fauna des Kantons Zürich. Käfer. Neue Denkschriften der allgemeinen schweizerischen Gesellschaft für die gesammten Naturwissenschaften 21: 1-240.

Favre E (1890) Faune des Coléoptères du Valais et des régions limitrophes. Zurcher, Furrer, 448 pp.

Focarile A (1984) Nuove ricerche sui popolamenti di coleotteri nel Ticino settentrionale - Campagne 1979-1982. Bolletino della Società Ticinese di Scienza Naturali 72: 7-55.*

Focarile A (1988) Ricerche sui Coleotteri del Parco alpino della Val Piora (Ticino, Svizzera). Bolletino della Società Ticinese di Scienza Naturali 76: 61-89.*

Focarile A (2003) Ecologia e faunistica degli insetti (particolarmente Coleotteri) nell'ecosistema alluviale della Val Bavona (Ticino, Svizzera). Rapporto Fondazione Valle Bavona, Cavergno, 85 pp.

Fontana P (1925) Contribuzione alla Fauna coleotterologica ticinese III. Bolletino della Società Ticinese di Scienza Naturali 19: 32-56.

Fontana P (1929) Note di Entomologia crepuscolare. Bolletino della Società Ticinese di Scienza Naturali 24: 121-127.

Fontana P (1947) Contribuzione alla fauna coleotterologica ticinese. Bolletino della Società Ticinese di Scienza Naturali 42: 16-94.

Freude H, Harde KW, Lohse GA (1967) Die Käfer Mitteleuropas. Band 7. Clavicornia. Goecke, Evers, Krefeld, 310 pp.

Freude H, Harde KW, Lohse GA (1979) Die Käfer Mitteleuropas. Band 6. Diversicornia. Goecke, Evers, Krefeld, 367 pp.

Frey-Gessner E (1900-1901) Souvenirs d'excursions d'un entomologiste dans le Val d'Anniviers. 1865-1900. Bulletin de la Murithienne 29-30: 66-72.*

Frey-Gessner E (1900) Liste des Coléoptères et des Lépidoptères intéressants notés par M. le chanoine E. Favre. Bulletin de la Murithienne 29-30: 73-77.*

Gaud A (1904) Binn. Course du 27 juillet au 1er août 1903. Bulletin de la Murithienne 33: 55-59.*

Gaud A (1905) Note entomologique. Bulletin de la Murithienne 34: 277-283.*

Gehrig J (1965) Neue Käferfunde im Allschwilerwald. Mitteilungen der Entomologischen Gesellschaft Basel 15(3): 41-43.*

Germann C (2010) Die Rüsselkäfer (Coleoptera, Curculionoidea) der Schweiz - Checkliste mit Verbreitungsangaben nach biogeografischen Regionen. Mitteilungen der Schweizerischen Entomologischen Gesellschaft 83: 41-118.
Germann C, Geiser M, Luka H, Sprecher E, Schatz I (2015) Käfer (Coleoptera) im Gebiet des Furkapasses, Kantone Uri und Wallis. Entomo Helvetica 8: 71-79.*

Gerstmeier R (1998) Checkered Beetles. Illustrated Key to the Cleridae of the Western Palaearctic. Magraf Verlag, Weikersheim, 241 pp.

Geis K-U (2001) Nochmals zum autochthonen Vorkommen von Tarsostenus univittatus (Rossi) (Col., Cleridae), zusammen mit Trogoxylon impressum (Com.) (Col., Lyctidae) in Südbaden. Mitteilungen der Entomologischen Vereins Stuttgart 36: 63-64.

Gfeller W (1987) Bemerkenswerte Käferfunde (Coleoptera) in der Schweiz, insbesondere im Tessin in den Jahren 1985-1987. Mitteilungen der Entomologischen Gesellschaft Basel 37(4): 183-186.*

Gfeller W (1992) Bemerkenswerte Käferfunde in der Schweiz, insbesondere in den Jahren 1988 bis 1992. 3. Beitrag zur Faunistik und Ökologie der Käfer in der Schweiz. Mitteilungen der Entomologischen Gesellschaft Basel 42(4): 163-168.

Gimmel ML, Bocáková M, Gunter NL, Leschen RAB (2019) Comprehensive phylogeny of the Cleroidea (Coleoptera: Cucujiformia). Systematic Entomology. https://doi.org/10.1111/syen.12338

Handschin E (1963) Die Coleopteren des schweizerischen Nationalparkes und seiner Umgebung. Ergebnisse der wissenschaftlichen Untersuchungen im schweizerischen Nationalpark. Band VIII. Lüdin, Liestal, 302 pp.

Hartmann K, Sprecher E (1990) Ein Beitrag zur Insektenfauna des Arlesheimer Waldes, unter besonderer Berücksichtigung der holzbewohnenden Käfer. Tätigkeitsberichte Naturforschende Gesellschaft Baselland 36: 75-124.*

Heer O (1841a) Die Kaefer der Schweiz, mit besonderer Berücksichtigung ihrer geographischen Verbreitung. Erster Theil, Dritte Lieferung. Petitpierre, Neuchatel, 79 pp.*

Heer O (1841b) Fauna coleopterorum helvetica. Pars 1. Turici: Impensis Orelii, Fuesslini et Sociorum, 652 pp.*

Herger P (1980) Die Insektenfauna des Hochmoores Balmoos bei Hasle, Kanton Luzern. V. Coleoptera (Käfer) - 1. Teil. Entomologische Berichte Luzern 4: 2-14.*

Herger P (1981a) Die Insektenfauna des Hochmoores Balmoos bei Hasle, Kanton Luzern. XII. Coleoptera (Käfer) - 2. Teil: Cantharoidea. Entomologische Berichte Luzern 6: 72-79.*

Herger P (1981b) Zur Insektenfauna des Siedereiteiches bei Hochdorf, Kanton Luzern. VI. Coleoptera (Käfer) - 1. Teil. Entomologische Berichte Luzern 5: 74-82.*

Herger P (1981c) Zur Insektenfauna der Umgebung des Brisen-Haldigrates, 1200-2400 m, Kanton Nidwalden. III. Coleoptera (Käfer) - 1. Teil. Entomologische Berichte Luzern 6: 64-71.*

Herger P (1982a) Zur Insektenfauna der Umgebung der Vogelwarte Sempach, Kanton Luzern. X. Coleoptera (Käfer) - 1. Teil. Entomologische Berichte Luzern 8: 68-82.*

Herger P (1982b) Zur Insektenfauna vom Pilatus-Kulm, 2060 m, Kanton Nidwalden. III. Coleoptera (Käfer) - 1. Teil. Entomologische Berichte Luzern 8: 48-56.*

Herger P (1983a) Käferfunde aus Littau, Kanton Luzern. Entomologische Berichte Luzern 9: 116-120.*

Herger P (1983b) Zur Insektenfauna der Umgebung von Baldegg, Kanton Luzern. Baldegg-Institut. III. Coleoptera 1 (ohne Staphylinidae und Curculionidae) (Käfer). Entomologische Berichte Luzern 10: 69-74 u. Anhang 81-88.*

Herger P (1983c) Zur Insektenfauna der Umgebung von Ettiswil, Kanton Luzern. III. Coleoptera 1 (ohne Staphylinidae und Curculionidae) (Käfer). Entomologische Berichte Luzern 10: 75-80 u. Anhang 81-88.* 
Herger P (1986) Zur Insektenfauna von Rigi-Kulm, 1600-1797 m, Kanton Schwyz. IV. Coleoptera 1: Carabidae - Scolytidae (ohne Staphylinidae). Entomologische Berichte Luzern 15: 1-11.*

Herger P (1987) Zur Insektenfauna von Gersau-Oberholz, Kanton Schwyz. IV. Coleoptera 1: Carabidae - Scolytidae (ohne Staphylinidae und Chrysomelidae). Entomologische Berichte Luzern 17: 1-19.*

Herger P (1989) Käferbeifänge aus 36 Borkenkäfer-Pheromonfallen im Forstrevier Rigi-Süd, 530-1620 m, Kanton Luzern, 1988 (Coleoptera). Entomologische Berichte Luzern 21: 33-44.*

Herger P (1990) Zur Insektenfauna des Urserentales, Furkastrasse 2000 m, Kanton Uri. IV. Coleoptera (Käfer). Entomologische Berichte Luzern 23: 23-28.*

Herger P (1991) Zur Insektenfauna von Obergütsch (500-600 m), Stadt Luzern. V. Coleoptera 3 (ohne Staphylinidae, Elateridae und Curculionidae). Entomologische Berichte Luzern 25: 27-40.*

Herger P (1992) Zur Insektenfauna vom Vogelmoos (775 m) bei Neudorf, Kanton Luzern. VI. Coleoptera 1 (ohne Staphylinidae, Elateridae, Chrysomelidae u. Curculionidae). Entomologische Berichte Luzern 28: 45-60.*

Herger P (1993) Zur Insektenfauna vom Airolo, Lüvina, 1200 m, Kanton Tessin. IV. Coleoptera 1: (ohne Nitidulidae, Cryptophagidae, Chrysomelidae). Entomologische Berichte Luzern 30: 13-30.*

Herger P (1994a) Die Käfersammlung von Josef Roos (1908-1992) Ein Beitrag zur Käferfauna der Talschaft Entlebuch, Kanton Luzern (Coleoptera). Entomologische Berichte Luzern 32: 1-12.*

Herger P (1994b) Käfer aus Lichtfallen im Engadin, 1840 - 2170 m, Kanton Graubünden. Mit Erstnachweis von Attalus alpinus Giraud (Malachiidae) für die Schweiz in diesem Jahrhundert (Coleoptera). Entomologische Berichte Luzern 32: 115-118.

Herger P (1995a) Käfer aus einer Lichtfalle bei Ins, Landwirtschaftliche Schule 430 m, Kanton Bern. - 1. Teil (Coleoptera). Entomologische Berichte Luzern 33: 57-66.*

Herger P (1995b) Käfer aus einer Lichtfalle bei Wädenswil, Sandhof, 518 m, Kanton Zürich (Coleoptera). Entomologische Berichte Luzern 34: 13-18.*

Herger P (1998a) Nachweise von Tenebroides fuscus (Goeze, 1777) in der hochmontanen Stufe des Napfgebietes (Coleoptera: Trogositidae). Entomologische Berichte Luzern 39: 105-106.

Herger P (1998b) Zur Insektenfauna des Kantons Schaffhausen (Hallau-Egg und Löhningen). III. Coleoptera (Käfer). Entomologische Berichte Luzern 39: 113-126.*

Herger P (1999a) Zur Insektenfauna vom Hochmoor Forenmoos, 970 m, Eigental, Kanton Luzern. IV. Coleoptera (Käfer). Entomologische Berichte Luzern 41: 1-16.*

Herger P (1999b) Zur Käferfauna im Entlebuch, Kanton Luzern: Käferfänge in Doppleschwand, Aebnetgüetli $850 \mathrm{~m}$ (Coleoptera). Entomologische Berichte Luzern 41: 17-22.*

Herger P (2002) Zur Insektenfauna vom Rüss-Spitz (Kanton Zug), 388 m, bei Maschwanden ZH. V. Coleoptera 1 (Käfer). Entomologische Berichte Luzern 47: 1-24.*

Herger P (2004) Zur Insektenfauna vom Hanenriet bei Giswil, 470 m, Kanton Obwalden. III. Coleoptera 1 (Käfer). Entomologische Berichte Luzern 51: 1-18.*

Herger P (2005) Zur Insektenfauna des Flachmoores Wauwilermoos, 498 m, Kanton Luzern. III. Coleoptera 1 (Käfer). Entomologische Berichte Luzern 53: 1-20.*

Herger P (2006) Zur Insektenfauna von Altdorf und Umgebung, Kanton Uri. 2. Reussdelta bei Seedorf, 435 m. IV. Coleoptera 2: Allgemeiner
Überblick und Artenliste 2. Teil (ohne Staphylinidae und Curculionidae). Entomologische Berichte Luzern 55: 1-20.*

Herger P (2007a) Zur Insektenfauna der Umgebung von Lauerz, Kanton Schwyz. 1. Sägel (455 m) und Schuttwald (480 m). VII. Coleoptera 2: Allgemeiner Überblick und Artenliste 2. Teil (ohne Staphylinidae und Curculionidae). Entomologische Berichte Luzern 57: 47-70.*

Herger P (2007b) Zur Insektenfauna der Umgebung von Lauerz, Kanton Schwyz. 2. Schwändi (650m). V. Coleoptera 2: Allgemeiner Überblick und Artenliste 2. Teil (ohne Staphylinidae und Curculionidae). Entomologische Berichte Luzern 57: 71-78.*

Herger P, Germann C (2015) Käfer (Coleoptera) vom östlichen Napfgebiet (Romoos und Umgebung), Kanton Luzern. Entomo Helvetica 8: 59-64.*

Herger P, Uhlig M (1990a) Zur Insektenfauna vom Fronalpstock (Kulm $1990 \mathrm{~m}$ und Oberfeld, $1860 \mathrm{~m}$ ), Kanton Schwyz. IV. Coleoptera (Käfer). Entomologische Berichte Luzern 24: 107-114.*

Herger P, Uhlig M (1990b) Zur Insektenfauna von Hospental, Kanton Uri. III. Coleoptera (Käfer). Entomologische Berichte Luzern 23: 15-22.*

Herger P, Germann C, Uhlig M, Vogel J, Geiser M, Kirejtshuk A (2015) Käfer aus Lichtfängen am Monte San Giorgio bei Serpiano, Kanton Tessin (Coleoptera). Entomo Helvetica 8: 89-96.*

Heyden von L (1863) Beitrag zur Coleopterenfauna des Ober-Engadins, insbesondere der Umgegend von St. Moritz. Jahresberichte der naturforschenden Gesellschaft Graubündens 8: 1-52.

Heyden von L (1864) Nachtrag zum Beitrag der Coleopterenfauna des Oberengadins, insbesondere der Umgegend von St. Moritz. Jahresberichte der naturforschenden Gesellschaft Graubündens 9: 1-16.

Heyden von L (1871) Supplement zum Beitrag der Coleopterenfauna des Ober-Engadins, insbesondere der Umgegend von St. Moritz. Jahresberichte der naturforschenden Gesellschaft Graubündens 16: 27-49.*

Hubenthal W (1916) Über Opilo germanus Chevrolat. Entomologische Blätter 12: 258-260.

Huber B, Büche B (2014) Vielfalt der Totholzkäferfauna im Urwald Scatlè, Breil/Brigels (Schweiz, Graubünden) (Coleoptera). Mitteilungen der Schweizerischen Entomologischen Gesellschaft 87: $311-326 . *$

Huber C, Kobel E (1994) Zum Vorkommen von Tenebroides fuscus (Goeze, 1777) in der Schweiz (Coleoptera, Trogositidae). Mitteilungen der Schweizerischen Entomologischen Gesellschaft 67: 1-5.

Hugentobler H (1959) Beitrag zur Kenntnis der Käferfauna des Thurgaus. Mitteilungen der Thurgauischen Naturforschenden Gesellschaft 38: 5-116.*

Hugentobler H (1966) Beitrag zur Kenntnis der Käfer der Nordostschweiz. Naturwissenschaftlichen Gesellschaft St. Gallen, 248 pp.*

Hunt H, Bergsten J, Levkanicova Z, Papadopoulou A, St. John O, Wild R, Hammond PM, Ahrens D, Balke M, Caterino MS, Gómez-Zurita J, Ribera I, Barraclough TG, Bocáková M, Bocák L, Vogler AP (2007) A comprehensive phylogeny of beetles reveals the evolutionary origins of a superradiation. Science 318: 1913-1916. https://doi. org/10.1126/science. 1146954

Jaccard H (1890) Catalogue des Coléoptères récoltés à Aigle et environs. Bulletin de la Murithienne 19-20: 21-60.

Jelínek J (2007) Biphyllidae [547-548]. In: Löbl I, Smetana A (Eds) (2007) Catalogue of Palaearctic Coleoptera, Vol. 4. Elateroidea Derodontoidea - Bostrichoidea - Lymexyloidea - Cleroidea - $\mathrm{Cu}-$ cujoidea. Apollo Books, Stenstrup, 935 pp.

Jörger JB (1914) Ein Beitrag zur Coleopteren-Fauna des Rigi. Mitteilungen der Schweizerischen Entomologischen Gesellschaft 12: 190-193.* 
Juillerat P, Bäumler B, Bornand C, Gygax A, Jutzi M, Möhl A, Nyffeler R, Sager L, Santiago H, Eggenberg S (2017) Checklist 2017 der Gefässpflanzenflora der Schweiz / de la flore vasculaire de la Suisse / della flora vascolare della Svizzera. Info Flora, 380 pp.

Kahlen M, Hellrigl K (1996) Ordnung Coleoptera-Käfer [393-511]. In: Hellrigl K (Ed.) Die Tierwelt Südtirols. Naturmuseum Südtirol, Bozen, $831 \mathrm{pp}$.

Kiesenwetter von H (1859) Kleinere Mittheilungen. Über die Fauna von Ragaz. Berliner Entomologische Zeitschrift 3: 339-345. https://doi. org/10.1002/mmnd.47918590307 *

Kiesenwetter von H (1861) Eine entomologische Excursion in das Wallis und nach dem Monte Rosa im Sommer 1861. Berliner Entomologische Zeitschrift 5: 360-395.

Killias E (1860) Zoologische Mittheilungen. 2. Insectenverzeichniss aus Puschlav. Jahresbericht der Naturforschenden Gesellschaft Graubündens 7: 102-108.

Köhler F (2000) Erster Nachtrag zum Verzeichnis der Käfer Deutschlands. Entomologische Nachrichten und Berichte, Dresden, 44(1): 60-84.

Köhler F (2011) 2. Nachtrag zum „Verzeichnis der Käfer Deutschlands“ (Köhler, Klausnitzer 1998) (Coleoptera). Entomologische Nachrichten und Berichte, Dresden, 55: 109-174, 247-254.

Köhler F, Klausnitzer B (1998) Entomofauna Germanica. Verzeichnis der Käfer Deutschlands. Entomologische Nachrichten und Berichte, Dresden, Beiheft 4: 1-185.

Kolibáč J (1992) Species of the genera Thanasimus Latreille and Korynetes Herbst in central Europe (Coleoptera, Cleridae). Acta entomologica Bohemoslovaca 89: 309-314.

Kolibáč J (1993) Observations on Ancyrona Reitter, 1876 with a key to Central European Trogositidae (Coleoptera, Trogositidae). Nachrichtenblatt der Bayerischen Entomologen 42(1): 16-22.

Kolibáč J (2007) Trogossitidae [364-366]. In: Löbl I, Smetana A (Eds) (2007) Catalogue of Palaearctic Coleoptera, Vol. 4. Elateroidea Derodontoidea - Bostrichoidea - Lymexyloidea - Cleroidea - $\mathrm{Cu}-$ cujoidea. Apollo Books, Stenstrup, 935 pp.

Kolibáč J (2013) Trogossitidae: A review of the beetle family, with a catalogue and keys. ZooKeys 366: 1-194. https://doi.org/10.3897/ zookeys.366.6172

Kolibáč J (2018) Icones insectorum Europae centralis. Coleoptera: Cleroidea: Phloiophilidae, Acanthocnemidae, Trogossitidae, Thanerocleridae, Cleridae. Folia Heyrovskyana, series B 33: 1-29.

Kolibáč J, Majer K, Švihla V (2005) Cleroidea. Beetles of the superfamily Cleroidea in the Czech and Slovak Republics and neighbouring areas. Clarion production, Praha, 186 pp.

Korge H (1960) Faunistische Neuigkeiten aus Deutschland: Opilo germanus. Entomologische Blätter 56: 185-186.

Lachat T, Pauli D, Gonseth Y, Klaus G, Scheidegger C, Vittoz P, Walter T (2010) Evolution de la biodiversité en Suisse depuis 1900. Avonsnous touché le fond? Zürich, Bristol-Stiftung; Bern, Stuttgart, Wien, Haupt, 433 pp.

Liberti G (1985) IV contributo alla conoscenza del genere Danacea Cast. (Col., Dasytidae). Descrizione del sottogenere nuovo Allodanacea e revisione delle specie italiane. Annali del Museo civico di Storia naturale « G. Doria » di Genova 85: 333-362.

Liberti G (1989) V contributo alla conoscenza del genere Danacea Cast. (Coleoptera, Dasytidae). Revisione delle specie italiane del II e del III gruppo. Entomologica Basiliensa 13: 279-302.
Liberti G (2009a) The Danacea of the Balkan Peninsula. A revision (Coleoptera Dasytidae). Memorie della Società entomologica italiana 88: 17-116. https://doi.org/10.4081/memorieSEI.2009.17

Liberti G (2009b) The Dasytidae (Coleoptera) of Sardinia. Zootaxa 2318: 339-385.

Liberti G (2012) A contribution to the knowledge of the european Trichoceble Thomson, 1859 (Coleoptera, Cleroidea, Dasytidae). Annali del Museo civico di Storia naturale « G. Doria » di Genova 104: 191-252.

Liberti G, Constantin R (2009) The Enicopus Stephens, 1830 species east of the Iberian peninsula: E. ater (Fabricius, 1898) and E. pilosus (Scopoli, 1763) (Coleoptera, Dasytidae). Annali del Museo civico di Storia naturale « G. Doria » di Genova 100: 293-321.

Liberti G, Constantin R (2011) Notes synonymiques et taxonomiques sur Dasytes gonocerus Mulsant, Rey, 1868, et D. incertus Schilsky, 1895 (Coleoptera, Cleroidea, Dasytidae). Bulletin de la Société entomologique de France 116 (2): 165-168.

Liberti G, Focarile A (2005) I Dasytidae del Cantone Ticino (Coleoptera, Cleroidea). Bolletino della Società Ticinese di Scienza Naturali 93: 19-39.

Linder A (1937) Beitrag zur Coleopteren-Fauna der Schweiz. Mitteilungen der Schweizerischen Entomologischen Gesellschaft 17: 172175.*

Linder A (1941) La collection de coléoptères du Chanoine Cerutti. Bulletin de la Murithienne 59: 129-134.

Linder A (1946) 2. Beitrag zur Coleopteren-Fauna der Schweiz. Mitteilungen der Schweizerischen Entomologischen Gesellschaft 20: 197-207.

Linder A (1953) 3. Beitrag zur Coleopteren-Fauna der Schweiz. Mitteilungen der Schweizerischen Entomologischen Gesellschaft 26(1): $63-71$.

Linder A (1967) Nachtrag zum Verzeichnis der Bündner Coleopteren von Dr. E. Killias. Jahresbericht der Naturforschenden Gesellschaft Graubünden 93: 78-109.

Linder A (1968) 4. Beitrag zur Coleopteren-Fauna der Schweiz. Mitteilungen der Schweizerischen Entomologischen Gesellschaft 41: 211-232.

Liniger E (1886) Ein Aufenthalt im Wallis. Mitteilungen der Schweizerischen Entomologischen Gesellschaft 7: 286-294.*

Lohse GA, Lucht WH (Eds) (1992) Die Käfer Mitteleuropas. 2. Supplementband mit Katalogteil. Goecke, Evers, Krefeld, 375 pp.

Löbl I (2007) Byturidae [546-547]. In: Löbl I, Smetana A (Eds) (2007) Catalogue of Palaearctic Coleoptera, Vol. 4. Elateroidea - Derodontoidea - Bostrichoidea - Lymexyloidea - Cleroidea - Cucujoidea. Apollo Books, Stenstrup, 935 pp. https://doi. org/10.1163/9789004260894

Löbl I, Smetana A (2007) Catalogue of Palaearctic Coleoptera, Vol. 4. Elateroidea - Derodontoidea - Bostrichoidea - Lymexyloidea Cleroidea - Cucujoidea. Apollo Books, Stenstrup, 935 pp. https:// doi.org/10.1163/9789004260894

Löbl I, Rolčík J, Kolibáč J, Gerstmeier R (2007) Cleridae [367-384]. In: Löbl I, Smetana A (Eds) Catalogue of Palaearctic Coleoptera, Vol. 4. Elateroidea - Derodontoidea - Bostrichoidea - Lymexyloidea Cleroidea - Cucujoidea. Apollo Books, Stenstrup, 935 pp. https:// doi.org/10.1163/9789004260894

Luka H, Nagel P, Feldmann B, Luka A, Gonseth Y (2009) Checkliste der Kurzflügelkäfer der Schweiz (Coleoptera: Staphylinidae ohne 
Pselaphinae). Mitteilungen der Schweizerischen Entomologischen Gesellschaft 82: 61-100.

Marggi W, Luka H (2001) Laufkäfer der Schweiz - Gesamtliste 2001 (Coleoptera: Carabidae). Checklist 2001, Carabidae of Switzerland. Opuscula Biogeographica Basileensia 1,37 pp.

Mayor A (2007a) Dasytidae, Malachiidae [388-454]. In: Löbl I, Smetana A (Eds) Catalogue of Palaearctic Coleoptera, Vol. 4. Elateroidea - Derodontoidea - Bostrichoidea - Lymexyloidea - Cleroidea - $\mathrm{Cu}$ cujoidea. Apollo Books, Stenstrup, 935 pp.

Mayor A (2007b) Phloiophilidae [363-364]. In: Löbl I, Smetana A (Eds) Catalogue of Palaearctic Coleoptera, Vol. 4. Elateroidea - Derodontoidea - Bostrichoidea - Lymexyloidea - Cleroidea - Cucujoidea. Apollo Books, Stenstrup, 935 pp.

Meier C, Sauter W (1989) Zur Kenntnis der Insektenfauna eines Auwaldreservates an der Aare bei Villnachern AG. Mitteilungen der Aaragauischen Naturforschenden Gesellschaft 32: 217-258.*

Michaud A (1937) Observations sur la Faune entomologique du Val d'Orvin. Bulletin de la Société Neuchâteloise des Sciences Naturelles 62: 84-98.*

Monnerat C, Chittaro Y, Sanchez A, Gonseth Y (2015a) Critères et procédure d'élaboration de listes taxonomiques nationales: le cas des Buprestidae, Cerambycidae, Lucanidae et Cetoniidae (Coleoptera) de Suisse. Mitteilungen der Schweizerischen Entomologischen Gesellschaft 88: 155-172.

Monnerat C, Chittaro Y, Sanchez A, Gonseth Y (2015b) Liste commentée des Lucanidae, Cetoniidae, Buprestidae et Cerambycidae (Coleoptera) de Suisse. Mitteilungen der Schweizerischen Entomologischen Gesellschaft 88: 173-228.

Mory CE (1894) Eine coleopterologische Sammelreise in Graubünden. Societas entomologica 8: 148-149, 162.*

Mory CE (1898) Liste der 1898 im Jouxthal gesammelten Coleopteren. Mitteilungen der Schweizerischen Entomologischen Gesellschaft 10: 469-473.*

Müller JA (1904) 3. Beitrag zur Coleopteren-Fauna der Kantone St. Gallen und Appenzell. Berichte der St. Gallischen Naturwissenschaftlichen Gesellschaft 10: 201-218.

Müller JA (1912) Verzeichnis der Käfer Vorarlbergs. Sonderabdruck aus dem 48. Jahresbericht des Landesmuseumsvereins Vorarlberg, Bregenz, 203 pp.

Niehuis M (2013) Die Buntkäfer in Rheinland-Pfalz und im Saarland. Gesellschaft für Naturschutz und Or nithologie Rheinland-Pfalz e.v. (GNOR), Mainz, 683 pp.

Pardo Alcaide A (1962) Malachiidae del Mediterráneo occidental (Coleoptera). Eos 38(2): 263-298.

Plata Negrache P (2009) Descripción de Ebaeus jperezvalcarceli, especie nueva de España y estudio de los Ebaeus del grupo collaris de la cuenca del Mediterráneo occidental (Coleoptera: Malachiidae). Boletín Sociedad Entomológica Aragonesa 45: 39-52.

Plata Negrache P (2012) Estudio de la Subfamilia Malachiinae Fleming en Andalucía. Fotocopias Campus, Domingo Pérez Batista, La Laguna, 203 pp.

Rätzer A (1888) Nachträge zur Fauna coleopterorum Helvetiae besonderes aus dem Gebiete des berner Seelandes, des Jura und der Walliseralpen. Mitteilungen der Schweizerischen Entomologischen Gesellschaft 8: 20-42.

Rehfous M (1955) Contribution à létude des Insectes des Champignons. Mitteilungen der Schweizerischen Entomologischen Gesellschaft 28: 1-106.*
Reibnitz J, Graf R, Coray A (2013) Verzeichnis der Ciidae (Coleoptera) der Schweiz mit Angaben zur Nomenklatur und Ökologie. Mitteilungen der Schweizerischen Entomologischen Gesellschaft 86: 63-88.

Rühl F (1887) Beitrag zur Coleopterenfauna des Averser-Thales. Societas entomologica 2: 90-91.*

Sanchez A, Chittaro Y (2017) Inventaire des Coléoptères saproxyliques des pinèdes du Val d'Anniviers (VS). Bulletin de la Murithienne 134: $21-34 .^{*}$

Sanchez A, Chittaro Y (2018) Liste commentée des Histeridae et Sphaeritidae de Suisse (Coleoptera, Histeroidea). Entomologische Blätter und Coleoptera 114: 335-352.

Sanchez A, Chittaro Y, Gonseth Y (2018) Préférences écologiques des coléoptères saproxyliques emblématiques de Suisse. Schweizerische Zeitschrift für Forstwesen 169(3): 158-165. https://doi.org/10.3188/ szf. 2018.0158

Sanchez A, Chittaro Y, Monnerat C (2015) Coléoptères nouveaux ou redécouverts pour la Suisse ou l'une de ses régions biogéographiques. Entomo Helvetica 8: 119-132.

Sanchez A, Chittaro Y, Monnerat C, Gonseth Y (2016) Les coléoptères saproxyliques emblématiques de Suisse, indicateurs de la qualité de nos forêts et milieux boisés. Mitteilungen der Schweizerischen Entomologischen Gesellschaft 89: 261-280.

Schacht W (1879) Contributions à la faune entomologique du Valais. III. Contribution à la faune des coléoptères du Valais. Bulletin de la Murithienne 9: 43-54.

Scherler P (1981) Notes coléoptérologiques. Bulletin Romand d'Entomologie 1: 17-22.*

Scherler P (1992) JORLOG, études entomologique des parcelles 06, 38, 39, 40, 41 et 42. Echantillonnage des Coléoptères. Bulletin Romand d'Entomogie 10: 59-80.*

Scherler P (1995) Les Coléoptères de la Grande Cariçaie (rive sud-est du lac de Neuchâtel). Bulletin Romand d'Entomologie 13: 31-54.

Scherler P, Sekaly V, Toumayeff G (1989) Coléoptères de la réserve du Bois de Chênes, dans la région de Ferreyres-Moiry. Bulletin Romand d)Entomologie 7: 11-29.*

Sprecher E, Luka H, Germann C, Luka A, Klausnitzer B, Graff P (2008) Käfer. [308-331]. In: Baur B, Billen W, Burckhardt D (Eds) Vielfalt zwischen den Gehegen: wildlebende Tiere und Pflanzen im Zoo Basel. Monographien der Entomologischen Gesellschaft Basel 3.

Stäger R (1951) Biologische Beobachtungen an Käfern im Wallis. Mitteilungen der Schweizerischen Entomologischen Gesellschaft 24: $125-128 . *$

Stierlin G (1863) Verzeichniss der während einer entomologischen Exkursion nach dem Engadin im Juni 1862 gesammelten Käfer. Mittheilungen der Schweizerischen Entomologischen Gesellschaft 1: 57-66.

Stierlin G (1863-1864) Zusammenstellung der durch Herrn Meyer-Dür in Tessin und Oberengadin beobachteten und eingesammelten Coleopteren. Mittheilungen der Schweizerischen Entomologischen Gesellschaft 1: 155-172.

Stierlin G (1875) Verzeichniss der gesammelten Käfer. Mitteilungen der Schweizerischen Entomologischen Gesellschaft 4: 465-468.*

Stierlin G (1880) Beiträge zur Kenntnis der Käferfauna des Kant. Wallis und der Dichotrachelus-Arten. Mittheilungen der Schweizerischen Entomologischen Gesellschaft 5: 541-551.*

Stierlin G (1883) Zweiter Nachtrag zur Fauna coleopterorum helvetica. Neue Denkschriften der schweizerischen Gesellschaft für die gesammten Naturwissenschaften 8(3): 1-98. 
Stierlin G (1898) Fauna coleopterorum helvetica. Die Käfer-Fauna der Schweiz nach der analytischen Methode. II. Theil. Bolli \& Böcherer, Schaffhausen, 662 pp.

Stierlin G (1900) Fauna coleopterorum helvetica. Die Käfer-Fauna der Schweiz nach der analytischen Methode. I. Theil. Bolli \& Böcherer, Schaffhausen, 667 pp.

Stierlin G (1906) Coleopteren-Fauna der Gegend von Schaffhausen. Mitteilungen der Schweizerischen Entomologischen Gesellschaft 11: $167-220$ * $^{*}$

Stierlin G, Gautard VV (1867) Fauna coleopterorum helvetica. Die Käfer-Fauna der Schweiz. Schaffhausen und Vevey, 372 pp.

Täschler M (1872) Beitrag zur Coleopteren-Fauna der Kantone St. Gallen und Appenzell. Berichte der Tätigkeiten der St. Galler Naturwissenschaftlichen Gesellschaft, 39-249.*
Tihelka E (2016) First record of Opilo germanus Chevrolat, 1843 (Coleoptera: Cleridae) from Italy with remarks on its distribution in Europe. Natura Sloveniae 18(2): 63-68.

Tronquet M (2014) Catalogue des Coléoptères de France. Perpignan, Association Roussillonnaise d'Entomologie, 1056 pp.

Tshernyshev SE (2009) Anthomalachius, a new genus of soft-winger flower beetles (Coleoptera, Malachiidae: Malachiinae). Zootaxa 2094: 23-35.

Uhlig M, Uhlig B (2006) Zur Käferfauna der Schweiz (Coleoptera ohne Staphylinidae). Entomologische Berichte Luzern 56: 1-20**

Walter T, Wolf M, Plattner M (2003) Holzbewohnende Käfer im Naturschutzgebiet Wildenstein. Mitteilungen der Naturforschenden Gesellschaften beider Basel 7: 263-285.*

Welti S (1998) Totholzabhängige Käfer (Coleoptera) und Totholzangebot im Sihlwald (Kt. Zürich). Diplomarbeit, Universität Zürich, 61 pp.* 